\title{
Effect of Pad Design and Bracket Manufacturing Method on Bracket Bond Strength
}

\author{
Dustin Osborne \\ dosborn1@mix.wvu.edu
}

Follow this and additional works at: https://researchrepository.wvu.edu/etd

Part of the Orthodontics and Orthodontology Commons

\section{Recommended Citation}

Osborne, Dustin, "Effect of Pad Design and Bracket Manufacturing Method on Bracket Bond Strength" (2021). Graduate Theses, Dissertations, and Problem Reports. 8130.

https://researchrepository.wvu.edu/etd/8130

This Thesis is protected by copyright and/or related rights. It has been brought to you by the The Research Repository @ WVU with permission from the rights-holder(s). You are free to use this Thesis in any way that is permitted by the copyright and related rights legislation that applies to your use. For other uses you must obtain permission from the rights-holder(s) directly, unless additional rights are indicated by a Creative Commons license in the record and/ or on the work itself. This Thesis has been accepted for inclusion in WVU Graduate Theses, Dissertations, and Problem Reports collection by an authorized administrator of The Research Repository @ WVU. For more information, please contact researchrepository@mail.wvu.edu. 


\title{
Effect of Pad Design and Bracket Manufacturing Method on Bracket Bond Strength
}

\author{
Dustin Osborne, D.D.S. \\ Thesis submitted \\ to the School of Dentistry \\ at West Virginia University \\ in partial fulfillment of the requirements for the degree of \\ Master of Science in \\ Orthodontics
}

\author{
Chris Martin, D.D.S., M.S. \\ Peter Ngan, D.M.D. \\ Susan Morgan, D.D.S. \\ Department of Orthodontics
}

Morgantown, West Virginia

2021

Keywords: SBS, MIM, ARI, milled, brazed, soldered, base

Copyright 2021 Dustin Osborne D.D.S. 


\title{
ABSTRACT \\ Effect of Pad Design and Bracket Manufacturing Method on Bracket Bond Strength
}

\author{
Dustin Osborne, D.D.S.
}

Objective: The objective is to determine if there is a significant difference in shear bond strength and debonding characteristics between one-piece metal brackets made by metal injection molding (MIM) and multi-piece metal brackets made by machining then soldering/brazing with differing pad designs.

Methods: A total of thirteen metal orthodontic brackets manufactured by 8 different companies were divided into two groups: one-piece MIM and multi-piece brackets. Each group consisted of 10 brackets for a total of 130 brackets. Brackets were bonded to stainless steel tubes with a universal bonding technique. Samples were tested on the universal Instron testing machine to measure the shear bond strength. Force required to debond each bracket was recorded. Brackets were observed after debond and an ARI score assigned. Data were analyzed using a two sample t-test.

Results: The mean shear bond strength and ARI scores for the entire study sample were 3.7 \pm 1.7 and 4.2 \pm 0.7 , respectively. The results of two sample $t$ test showed that there was no significant difference between the one-piece MIM brackets $(3.5 \pm 1.7)$ and the multi-piece brackets $(4.0 \pm 1.8)$ in mean shear bond strength $(\mathrm{t}(128)=1.33, \mathrm{p}=0.18)$. But the mean of ARI scores of the multipiece brackets $(4.4 \pm 0.7)$ was significantly higher than the one-piece MIM brackets $(4.0 \pm 0.7)$ $(\mathrm{t}(128)=3.5, \mathrm{p}=0.006)$.

Conclusion: One-piece metal orthodontic brackets manufactured by MIM and multi-piece brackets manufactured by machining then soldering/brazing exhibit similar shear bond strengths. However, one-piece brackets showed a significantly lower ARI score than multi-piece brackets, indicating a higher percentage of adhesive left on the one-piece bracket surface after debond. 


\section{DEDICATION}

To Mom, Thank you for being a constant source of love and support. I never could have made it anywhere in life without you. I appreciate all your hard work and never-give-up attitude. You've played an essential role in shaping me into the man I am today.

To Dad, Thank you for always pushing me to be my best and working hard to give the best childhood for Adam and I. You taught me that character counts and sometimes all you need to fix the problem is a bigger hammer. Thank you for the life you have given me.

To Adam, Thank you for being the best brother I could ask for and our parents second favorite son. You've inspired me to work hard and challenge myself in more ways than you know. You set a prime example for me and a tough act to follow as a younger brother. Without your lead, I would not be the person I am today.

To Dave and Josh, Thank you guys for being my family and best friends. You've helped me through the worst of times and been there to celebrate the best of times. I appreciate all the time we've shared and hope to have many more adventures in the future.

To Bill and Jules, Thank you guys for being great friends and all your support. I will always consider you guys to be a part of my extended family. I can't wait for all of our future trips and incredible experiences together.

To Dessie, Thank you for everything you've done for me over the years and how much you've given me. Your love and support means the world to me. I couldn't have done any of this without you.

To Cindy, Thank you for being an inspiration and constant source of love and support. I appreciate all you've done for me. I'm eternally grateful for the home gym you provided, as it has been a necessary source of stress release in 2020 .

To Clarence and Linette, Thank you for adopting me as your second son for many summers and allowing me to run wild with your boys. I grew extensively in those times and was able to make memories that will last a lifetime.

To Tom, Thank you for being a great friend who's always willing to listen and advise me when life throws its' curveballs. Your support has been incredible.

To Queso, Thank you for being a great friend and setting such a high academic bar for me. I probably would have given up and may not have pushed myself to the next level then the next level then the next level without you.

To Max, Thank you for being a great friend and always ready to have a good time. I appreciate your continued support and look forward to future adventures. 
To Cheese, Thank you for being a friend, wildcard and a great source of entertainment. I'm happy to have you as a friend and can't wait to see what life has in store for you.

To Alex, Thank you for being a great friend to me. I don't think I could've made it through dental school without your friendship and support. I wish you all the best in your future endeavors and know you will be successful no matter where you go.

To Sarah, Thank you for being such a great friend. I definitely couldn't have made it through residency without you. I appreciate your support, positive attitude and fun spirit. I wish you all the best as you navigate the world of private practice ownership. 


\section{ACKNOWLEDGMENTS}

Committee Members, Thank you for advising me and participating in my thesis project.

Michele, Thank you for always finding room for me to come over and shadow during dental school. I appreciate your care and support and always making my life a little easier each day.

Marsha, Thank you for being a great person and great support. You taught me far more than you'll ever know. I appreciate everything you did for me.

Dr. Dan Joseph, Thank you for the research project idea. Your giving spirit and amazing generosity is inspirational. I appreciate all that you did for myself and my family. Thank you for everything.

Vince Kish, Thank you for helping with my project. I appreciate your patience with the Instron machine which was last updated in the 1800's.

Jun Xiang, Thank you for being the statistician on my research project. I could have never interpreted the results without you.

Residents, Thank you for what you all have taught me. I've learned so much and been able to make many changes to who I am professionally and personally because of you all.

Dr. Ronnie Sparks, Thank you for teaching me everything you know and allowing me to bother you in your practice. Thank you for being a friend and mentor, always advising me on my toughest decisions. Your knowledge is inspiring and I hope for the worlds' sake you're able to teach more as time goes on. Future residents depend on your knowledge and guidance. You're an amazing example of what orthodontists should strive to be.

Dr. Larry Kalaskey, Thank you for being a great teacher, friend and mentor. I always appreciate your generosity and support. I appreciate you letting me come to shadow in your practice and showing me how to be successful. You've shown me it's possible to be a great orthodontist and still have an incredible life outside of work. Work hard, play hard.

Dr. Dan Foley, Thank you for opening your home to us residents and always showing us a fun and exciting time. Thanks for the opportunity to see that there is a life after residency and reminding us all why we got into the profession in the first place. Can't wait to take a ride in the Foley Defense jet. 


\section{TABLE OF CONTENTS}

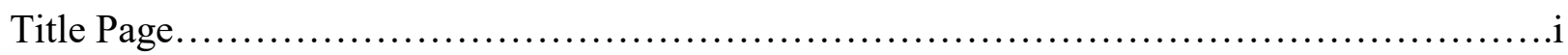

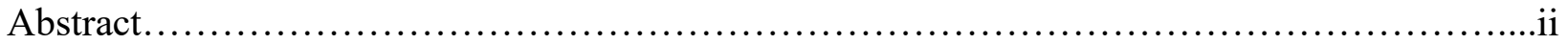

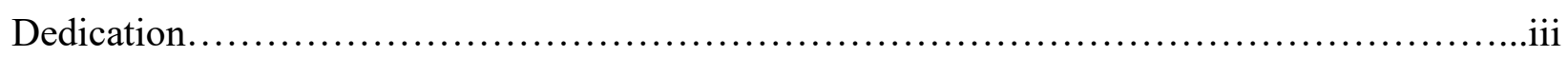

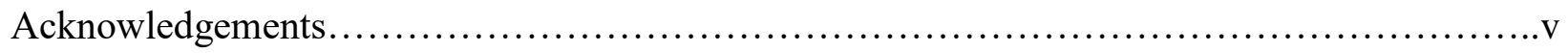

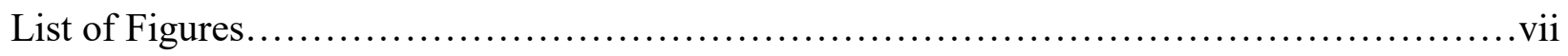

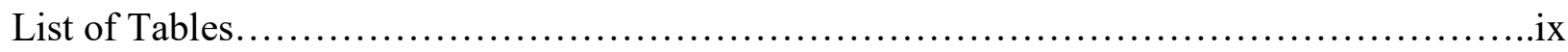

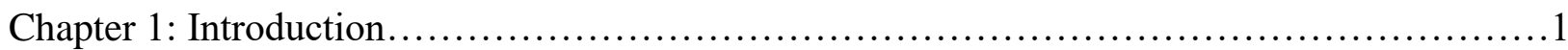

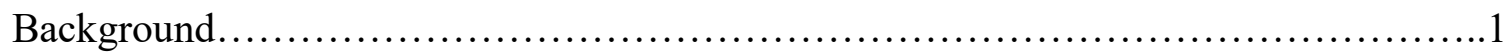

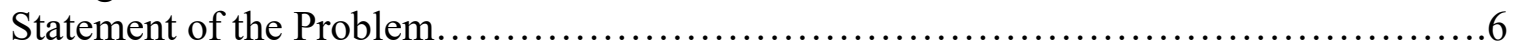

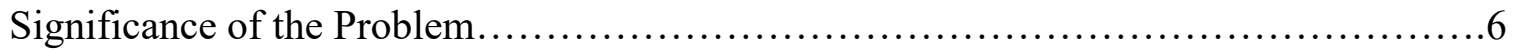

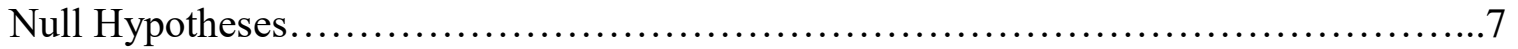

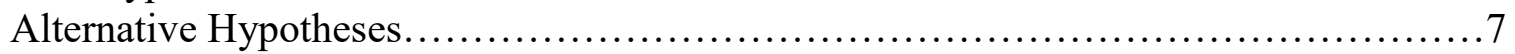

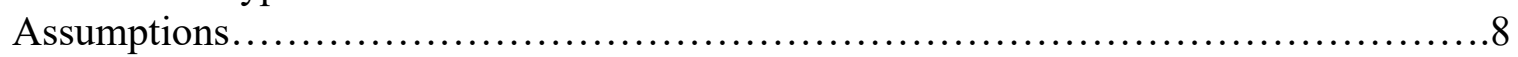

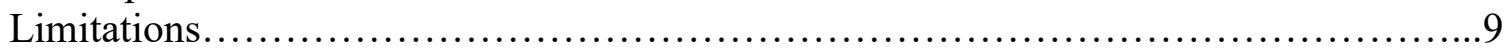

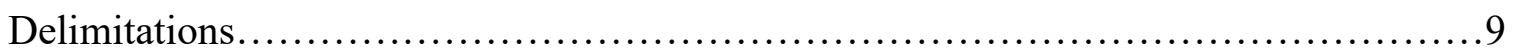

Chapter 2: Review of the Literature...................................................... 10

Chapter 3: Materials and Methods...........................................................

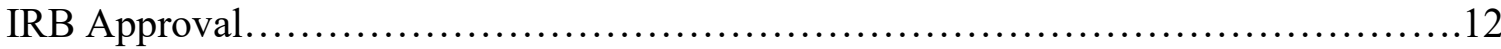

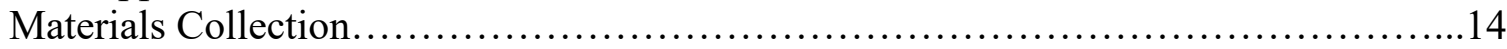

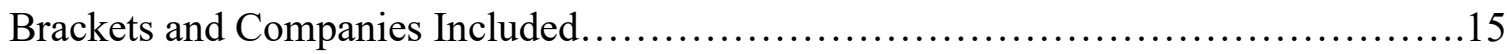

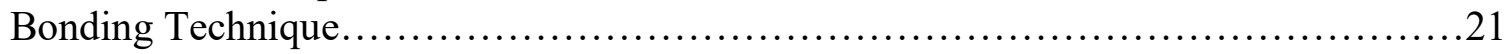

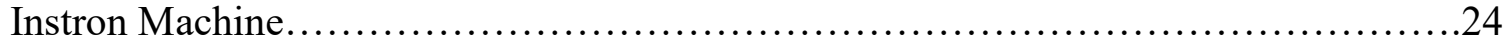

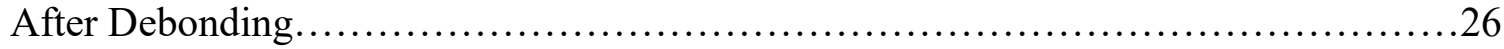

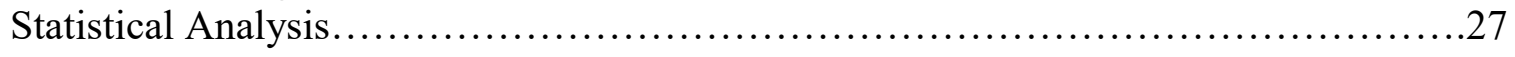

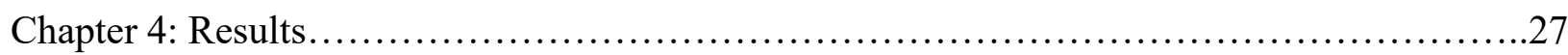

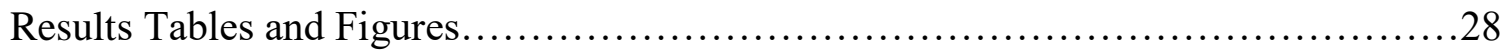

Null Hypotheses Testing.........................................................

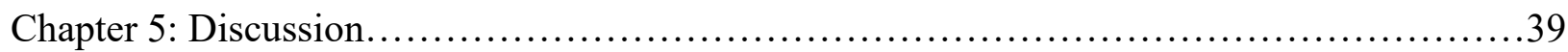

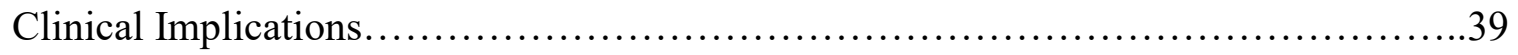

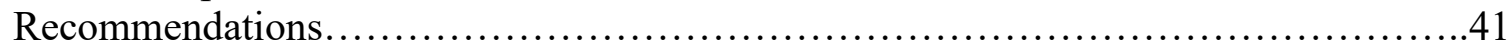

Chapter 6: Summary and Conclusion....................................................42

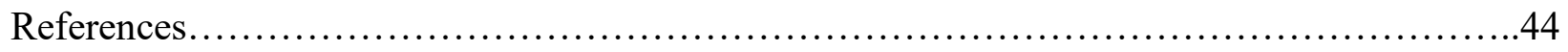




\section{LIST OF FIGURES}

Figure 1: Twin Bracket Anatomy.................................................

Figure 2: MIM Process............................................................. 5

Figure 3: 3M Miniature Twin Bracket............................................ 15

Figure 4: 3M Victory Series LP Bracket....................................... 15

Figure 5: American Orthodontics Master Series Bracket................................. 16

Figure 6: Forestadent Mini Mono Bracket......................................... 16

Figure 7: Forestadent Mini Sprint II Bracket..................................... 17

Figure 8: Rocky Mountain Orthodontics Integra Bracket................................17

Figure 9: Dynaflex MTX Bracket.................................................. 18

Figure 10: Dynaflex Atlas Mini Bracket.......................................... 18

Figure 11: Dentaurum Discovery Smart Bracket......................................19

Figure 12: Dentaurum Equilibrium II Bracket........................................ 19

Figure 13: GC Legend Medium Bracket...........................................20

Figure 14: Ormco Mini Diamond Twin Bracket.......................................20

Figure 15: Ormco Mini Twin Bracket...............................................21

Figure 16: Mounted Brackets and Mounting Jig.....................................23

Figure 17: Brackets Mounted to Stainless Steel Tube.....................................23

Figure 18: Brackets Bonded to Tube in Mounting Jig..................................24

Figure 19: Instron Machine and Debonding Apparatus.................................25

Figure 20: T-test of Shear Bond Strength........................................... 34

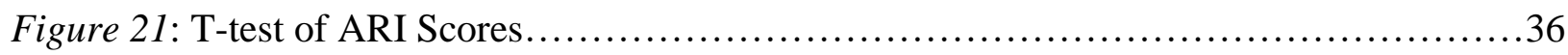

Figure 22: Wilcoxon Distribution of Shear Bond Strength.................................. 37 


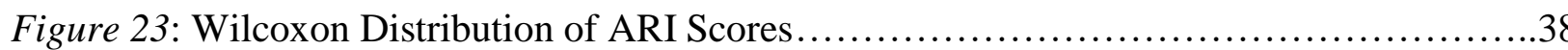




\section{LIST OF TABLES}

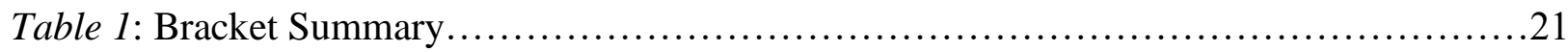

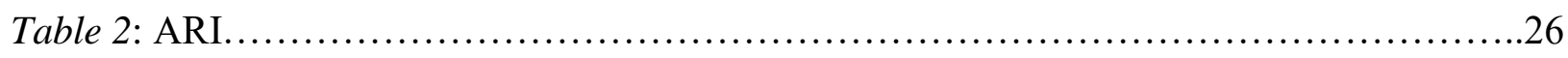

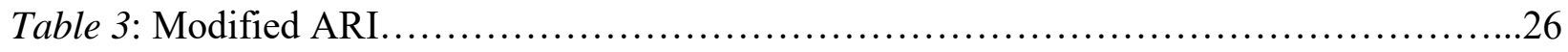

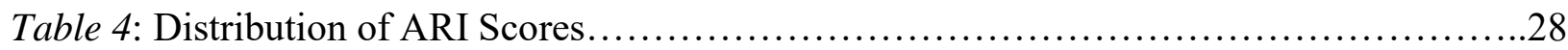

Table 5a: Moments for ARI scores of multi-piece brackets...............................28

Table 5b: Basic Measurements for ARI Scores of Multi-Piece Brackets......................28

Table 5c: Extreme Observations for ARI Scores of Multi-Piece Brackets.......................29

Table 6a: Moments for SBS of Multi-Piece Brackets......................................29

Table 6b: Basic Measurements for SBS of Multi-Piece Brackets.............................29

Table 6c: Extreme Observations for SBS of Multi-Piece Brackets..............................30

Table 7a: Moments for ARI scores of One-piece brackets................................. 30

Table 7b: Basic Measurements for ARI Scores of One-Piece Brackets.........................30

Table 7c: Extreme Observations for ARI Scores of One-Piece Brackets........................31

Table 8a: Moments for SBS of One-piece brackets........................................ 31

Table 8b: Basic Measurements for SBS of One-Piece Brackets................................ 31

Table 8c: Extreme Observations for SBS of One-Piece Brackets.............................32

Table 9a: T-test Values for Measurements of Shear Bond Strength...........................33

Table 9b: T-test Values for Differences of Shear Bond Strength...............................33

Table 9c: T-test Values for Variances of Shear Bond Strength...............................33

Table 9d: T-test Values for Equality of Variances of Shear Bond Strength......................33

Table 10a: T-test Values for Measurements of ARI Scores....................................... 
Table 10b: T-test Values for Differences of ARI Scores.

Table 10c: T-test Values for Variances of ARI Scores.......................................

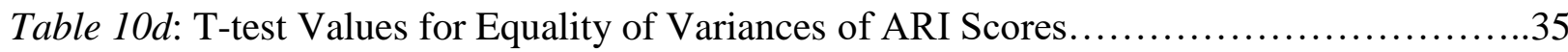




\section{Chapter 1: Introduction}

\section{Background}

Correction of malocclusions has been in practice for hundreds of years. Orthodontists move teeth to an ideal position within their respective bones for form and function, not just esthetics.

Malocclusions have been classified into three categories; class one, two and three. These classes are based solely on the relationship between the upper and lower permanent first molars. In a class one malocclusion, the mesiobuccal cusp tip of the upper first molar occludes directly with the buccal groove of the mandibular first molar; although the molar relationship is ideal, the malocclusion may be in the form of crowding, spacing, deep-bite, etc. In a class two malocclusion, the mesiobuccal cusp tip of the upper first molar falls anterior to the buccal groove of the mandibular first molar. Finally, in a class three malocclusion, the mesiobuccal cusp tip of the upper first molar occludes posterior to the buccal groove of the mandibular first molar. The causes of each type of malocclusion may be attributed to dental, skeletal or a combination of discrepancies. Not every malocclusion requires orthodontic intervention; that will be dependent of a barrage of other factors. The prevalence of class one malocclusions is predominant, followed by class two, then class three $12,16,18-21$.

Many different appliances may be used in treating malocclusions. Orthodontists have traditionally used metal brackets bonded to the facial/buccal surface of a tooth for movement; at least since the introduction of composite bonding materials that allow for adequate bonding strength of metal to enamel. Brackets have evolved over time in shape, size, material, etc. to meet the needs of practicing orthodontists. Many factors must be considered when making improvements to bracket design; patient comfort, style, durability, user-friendliness and strength are of utmost importance. With the introduction of clear aligners, orthodontists now have more 
choices than ever for modality of moving teeth. Clear aligners have come to prominence with the introduction of Invisalign ${ }^{\mathrm{TM}}$ in $1988^{23}$. This, however, is just another tool for the orthodontists' tool belt when deciding what method is best for treating a particular patient. Ultimately, it comes down to provider preference. With so many options to choose from, its important that no matter which modality is selected, both the patient and doctor have the most pleasant experience possible. A contributing factor to dissatisfaction in orthodontic treatment is frequency of unplanned emergency visits. In utilizing traditional metal brackets, emergency visits are commonly caused by brackets debonding from the tooth surface ${ }^{1,6-11}$. This phenomenon can be caused by many factors such as amount of orthodontic force, intraoral age of bracket, patient habits, etc ${ }^{3}$. Less commonly, the bracket itself may break, causing more patient discomfort and necessitating a more complex replacement procedure ${ }^{22}$. These are costly for both patient and provider in time and expense. To minimize these emergencies, selecting a product that has the best chance of remaining in place and in one piece for the duration of treatment is beneficial to all. With this knowledge in mind, orthodontic companies have developed different manufacturing techniques that may promise greater bracket strength and decreased debonding frequency.

Modern orthodontic brackets began with the introduction of the edgewise bracket by Dr. Angle in 1928. Edgewise simply refers to the orientation of the wire when engaged in the bracket. This bracket was narrow in the mesiodistal direction with only a single set of tie wings. The tie wings are the portion of the bracket that allow for placement of an "o-tie" or ligature to force wire engagement within the bracket itself. An additional set of tie wings was later added to orthodontic brackets, developing the "twin" bracket, which is still the most common used orthodontic bracket style today, according to Iijima. This twin bracket design was introduced by 
Swain and allowed for better tip, rotation and mesial-distal root movement control. The bracket had to become larger misodistally to accommodate the second set of tie wings. Early twin bracket design consisted of multiple pieces later joined to form a single unit. Figure 1 shows the standard design of a multi-piece orthodontic twin bracket. The tie wings contain the slot which receives the orthodontic wire and are located on the right and left sides of the profile in the figure. The brazing layer joins the profile to the base of the bracket. A second brazing layer may be needed to join the retentive mesh to the other side of the base.

Bracket bases can have mesh or non-mesh retentive features added. For mesh bases, different gauge wires are used for larger or finer retentions areas. Wire gauges range from 40100, with 100 gauge being the finest mesh size to accommodate a 155-micron particle size filler according to Gange. Mesh may be attached to the bracket base by welding or brazing. Welding is less commonly used today due to compromised mesh integrity, wire fracture, and production of stress concentrated areas leading to adhesive fracture. In contrast, during brazing, mesh integrity is maintained. During brazing, the filler is melted at temp above 450 degrees and below melting point of metal to be joined. The brazing layer typically consists of silver, gold or non-precious alloys. Additional means of added retention can come from etching, silanation, surface activation or a combination. Etching creates surface roughness that increases area for bonding. Silanation uses a silane-coupler to increase surface wetness for increased bonding area. Surface activation is a process of electrochemical cleaning to remove debris before bonding.

Each component of the bracket may be made by different manufacturing techniques; machining, milling, casting or molding. 


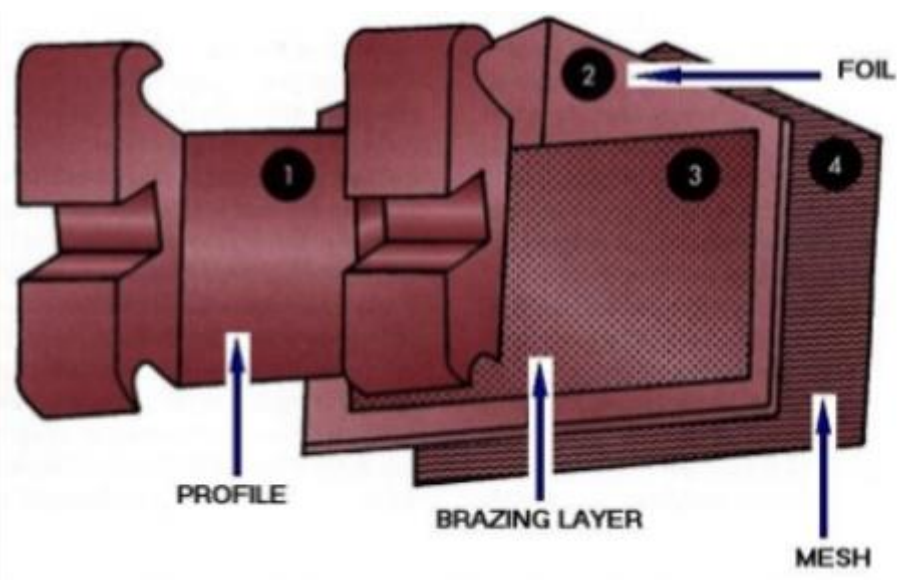

Figure 1: Twin Bracket Anatomy

The adherence joints create inherent points of weakness within the bracket and are more likely sites for bracket failure. The process of making multi-piece brackets is costly as each component is made separately and joined later. Components made by casting of milling are subject to 50$90 \%$ materials loss during the manufacturing process. To combat weak joints and introduce a less expensive manufacturing process, metal injection molding (MIM) has been recently implemented in fabricating orthodontic brackets. MIM is the process of liquifying metal alloy and injecting them into premade bracket molds (Figure 2). 
FIGURE 1.

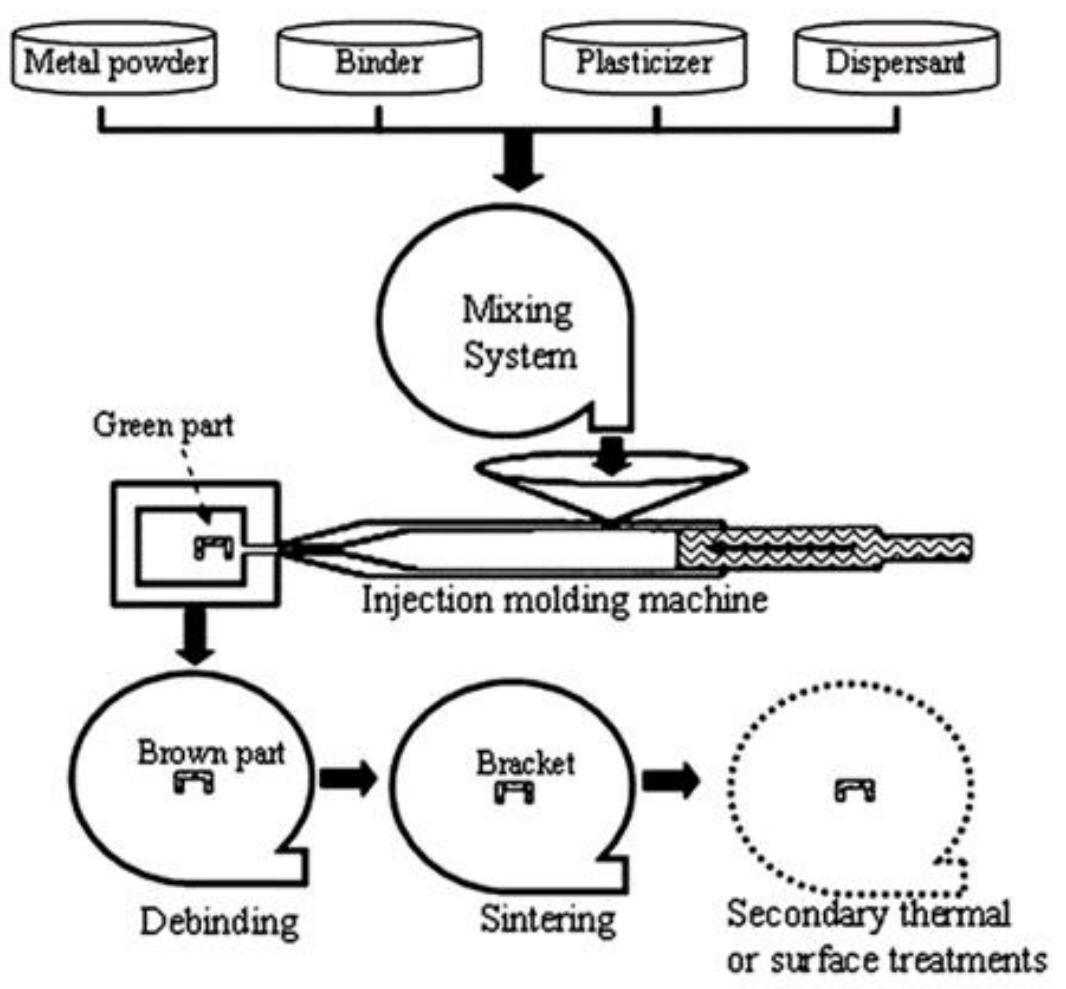

Figure 2: MIM process

Brackets are made larger initially to compensate for porosity shrinkage that occurs in the steps of sintering and debinding after the molding is complete. Utilization of MIM in orthodontic bracket manufacturing has allow for a truly one-piece bracket with built-in retentive features and no weak spots from brazing or soldering. This process does come with its' downsides; for example, weak spots may no longer exist within the bracket, but the bracket as a whole may be weaker and therefore more likely to experience breakage at any given point. This weakness comes from remaining porosity after shrinkage to final size has occurred.

The objective of this study is to determine the relative durability, shear bond strength and characteristics of bond failure of one-piece MIM brackets compared to multi-piece brackets manufactured by casting, milling, MIM, or a combination with different bracket base designs. 


\section{Statement of the Problem}

Do one-piece MIM metal brackets have the same in-vitro shear bond strength as multi-piece milled/machined metal brackets bonded to standardized stainless steel utilizing a common bonding technique?

Is there an effect of bracket pad design on shear bond strength of one-piece MIM metal brackets vs. multi-piece milled/machined metal brackets?

What are typical bracket debonding findings?

\section{Significance of the problem}

Traditional bracket manufacturing methods are costly and inefficient. As a potential alternative, brackets manufactured by MIM are more cost efficient. The MIM manufacturing technique holds the technological advantage and promises to be area of great research as new methods for fabrication develop. For now, differences in strength exist between multi-piece cast/milled brackets and one-piece MIM brackets due largely to porosities left after the MIM process. Traditionally, two-piece brackets yield greater overall strength and perform better in lab tests. This project will provide a true comparison between one-piece and multi-piece brackets. The aim will be to determine the manufacturing process that yields the greatest strength and will likely be the most reliable bracket to use in orthodontic procedures. The results of this study may be used to further develop and improve the MIM technique to eventually obtain similar strength to brackets made by casting or milling. More research will be required, as this will be an in vitro study. Similar future studies will need to be conducted in vivo to give more accurate results. Another aim of this study will be to act as a pilot research project for an in vivo experiment, narrowing the field to comparisons of the best tested brackets in vitro. 


\section{Null Hypotheses}

1. There is no significant intergroup difference in shear bond strength between one-piece brackets manufactured by the MIM process and multi-piece brackets with components manufactured by either MIM, milling or casting process

2. There is no significant intragroup difference in shear bond strength between different multi-piece brackets with components manufactured by MIM, milling, or casting.

3. There is no significant intragroup difference in shear bond strength between different one-piece brackets made by MIM.

4. There is no significant intergroup difference in shear bond strength between one-piece or two-piece brackets manufactured by MIM, casting, or milling and bracket pad design/retentive features (ex. Micro-etching, photo-etching, addition of mesh, etc.)

5. There is no significant intragroup difference in shear bond strength between one-piece brackets made by MIM and bracket pad design/retentive features.

6. There is no significant intragroup difference in shear bond strength between multi-piece brackets made with components manufactured by MIM, milling, or casting and bracket pad design/retentive features.

7. There is no significant difference in ARI score between one-piece MIM metal brackets and multi-piece brackets with components manufactured by MIM, milling, or casting.

\section{Alternative Hypotheses}

1. There is a significant intergroup difference in shear bond strength between one-piece brackets manufactured by the MIM process and multi-piece brackets with components manufactured by either MIM, milling or casting process 
2. There is a significant intragroup difference in shear bond strength between different multi-piece brackets with components manufactured by MIM, milling, or casting.

3. There is a significant intragroup difference in shear bond strength between different onepiece brackets made by MIM.

4. There is a significant intergroup difference in shear bond strength between one-piece or two-piece brackets manufactured by MIM, casting, or milling and bracket pad design/retentive features (ex. Micro-etching, photo-etching, addition of mesh, etc.)

5. There is a significant intragroup difference in shear bond strength between one-piece brackets made by MIM and bracket pad design/retentive features.

6. There is a significant intragroup difference in shear bond strength between multi-piece brackets made with components manufactured by MIM, milling, or casting and bracket pad design/retentive features.

7. There is no significant difference in ARI score between one-piece MIM metal brackets and multi-piece brackets with components manufactured by MIM, milling, or casting.

\section{Assumptions}

1. Operator has been calibrated to place brackets in the exact same position with the exact same amount of pressure.

2. Bonding technique is executed in the same manner for all brackets.

3. Same amount of bonding material is applied to each bracket in the same manner in sufficient amount to retain bracket to stainless steel mounting tube.

4. No contamination of brackets or bonding medium due to the in vitro nature of this study. 
5. Bracket base dimensions are assumed to be correctly reported by manufacturing company.

\section{Limitations}

1. Operator error will exist in placing brackets in the exact same position with the same amount of pressure.

2. Operator error in exactness of bonding technique between all brackets; slight variations will exist.

3. Operator error in apply the same amount of bonding material in the same manner to each bracket; slight variations will exist.

4. Contamination may exist due to in vitro nature of study completed in non-sterile environment.

5. Instron machine will simulate peel and shear force instead of pure shear force.

6. Operator error in verifying bracket measurements .

7. Discrepancies in ARI readings.

\section{Delimitations}

1. Single operator to reduce operator error between multiple operators calibrated differently.

2. Use of custom jig in bracket placement.

3. Use of adjustable base jig to ensure parallelism of applied force by Instron.

4. Two ARI readings taken at two week intervals to assess intraobserver accuracy. 


\section{Chapter 2: Review of the Literature}

The orthodontic bracket acts as the bridge of energy transfer from the archwire to the teeth. The original edgewise bracket was introduced by Dr. Angle in 1928. This bracket design is narrow and has only one set of tie-wings; one incisal wing and one gingival wing. The narrowness of each bracket allows for the use of lighter forces due to a long interbracket distance. Bracket styles have evolved and new, more widely used designs have been introduced. The twin bracket design, developed by Swain, has two sets of tie-wings, which allow for better tip, rotation and mesial-distal root movement control. The twin style bracket is the most widely used orthodontic bracket style today ${ }^{5}$. Bracket bonding techniques have evolved as well, creating s stronger adherence of the bracket to the tooth surface. Acid etching was introduced by Buonocore in the 1950s and direct bracket-enamel bonding was introduced by Newman in 1960s. Standard bracket design consists of a stainless steel bracket comprised of a base, slot and wings. In an effort to maximize the bracket-enamel bond and bracket strength, variations in bracket base design and bracket manufacturing techniques have been explored. Some differences in bracket base design to increase bond strength include adding a layer of mesh, milling undercuts, sandblasting, and chemically etching. Bracket wings and bases are traditionally made with stainless steel; types 303, 304, 316 and 17-4PH being most widely used ${ }^{2}$. A combination of desirable mechanical properties, corrosion resistance, and biocompatibility determine which stainless steel will be used. Some stainless steels, type 2205, do not contain nickel, which may be selected for use in patients with a metal allergy ${ }^{5}$. The wings and bases of brackets are made separately by casting and milling procedures. They are then soldered together. These processes may present many disadvantages. Both methods are inefficient and produce excessive waste; casting waste comes from sprue and runner material, while milling waste comes from material removed during the 
machining process. Soldering of the two components is achieved with an alloy filler material. Differences in chemical composition of the stainless steel components will determine the solderability of the parts, however most stainless steels are compatible with soldering alloys containing silver, gold, copper or nickel. Silver and gold based solders were used originally, but each have shown corrosive properties and questionable mechanical characteristics. No brazing alloys available can fulfill the corrosion resistance, compatibility and strength requirements of a good solder ${ }^{24}$. A more recent development in bracket manufacturing is by metal injection molding (MIM). This process requires metallic powders mixed with an organic binder to form a homogeneous mixture that can be injected into a mold and form the final desired shape. This method is less expensive than milling and casting due to a reduction of waste materials and eliminates the possibility of corrosion or bond failure at the wing-based interface. This method, however, is not without compromise. The MIM process is imprecise as the initial size of the injected shape is subsequently reduced by $20 \%$ in all dimensions as a result of the firing process. This process may also leave porosities in the final product that reduce the overall strength of the bracket. Studies using the Vickers hardness scale have shown MIM brackets ranging from 154287 hardness as compared to a 400 hardness for conventional stainless steel brackets. This difference could influence mechanical properties and wear resistance for MIM brackets ${ }^{2,4,25}$. 


\section{Chapter 3: Materials and Methods}

\section{IRB Approval}

Approval for exempt research was obtained from the Institutional Review Board of West

Virginia University (\# 1910741211) prior to the start of this study. 


\section{WestVirginiaUniversity. \\ Office of Research Integrity and Compliance \\ 886 Chesinut Ridge Road Norgantown, WV 26506}

\section{Acknowledgement of Not Human Subjects Research}

$10 / 11 / 2019$

To: Chris Martin

From: WVU Office of Research Integrity \& Compliance

Protocol Type: NHSR / Flex

Approval Date: $10 / 11 / 2019$

Submission Type: Initial

Expiration Date: 10/10/2024

Funding: N/A

WVU Protocol \#: 1910741211

Protocol Title: Effect of Bracket Pad Design and Bracket Manufacturing on Bond Strength

The West Virginia University Institutional Review Board has determined that your protocol submission of protocol 1910741211 does not meet the definition of human subjects research, as provided in 45 CFR 46. This determination was based on the following:

- Research means a systematic investigation designed to develop or contribute to generalizable knowledge. Most case reports and most oral histories are not generalizable and, therefore, not research. Many classroom projects, if not intended to be published, are also not considered research. Many quality improvement or program evaluation studies are not research.

The following documents were reviewed and acknowledged for use as part of this submission. Only the documents listed below may be used in the research. Please access and print the files in the Notes \& Attachments section of your approved protocol.

WVU IRB acknowledgement of protocol 1910741211 will expire on 10/10/2024. 
If the study is to continue beyond the expiration date, a new application must be submitted to the WVU IRB. It is your responsibility to submit your protocol for review.

The Office of Research Integrity and Compliance will be glad to provide assistance to you throughout the research process. Please feel free to contact us by phone, at 304.293 .7073 or by email at IRB@mail.wvu.edu.

Sincerely,

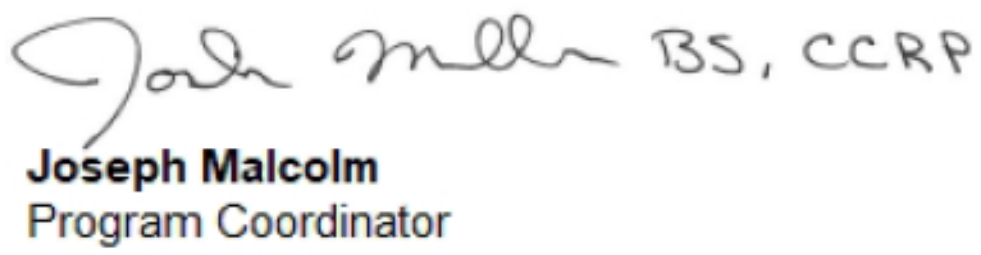

\section{Materials Collection}

Several top orthodontic companies were contacted to determine the method in which their brackets are manufactured. Companies that manufacture brackets using both MIM and milling/machining techniques were asked to supply 10 upper lateral incisor brackets made by each process. Companies that manufacture brackets using only one of the two manufacturing methods were asked to supply 10 upper lateral incisor brackets made by that process.

Upper lateral brackets were selected due to a reasonable consistency in two dimensional size (inciso-gingival height and mesio-distal width) and bracket pad contour. 


\section{Brackets and Companies Included}

\section{1. $3 \mathbf{M}$ (Unitek)}
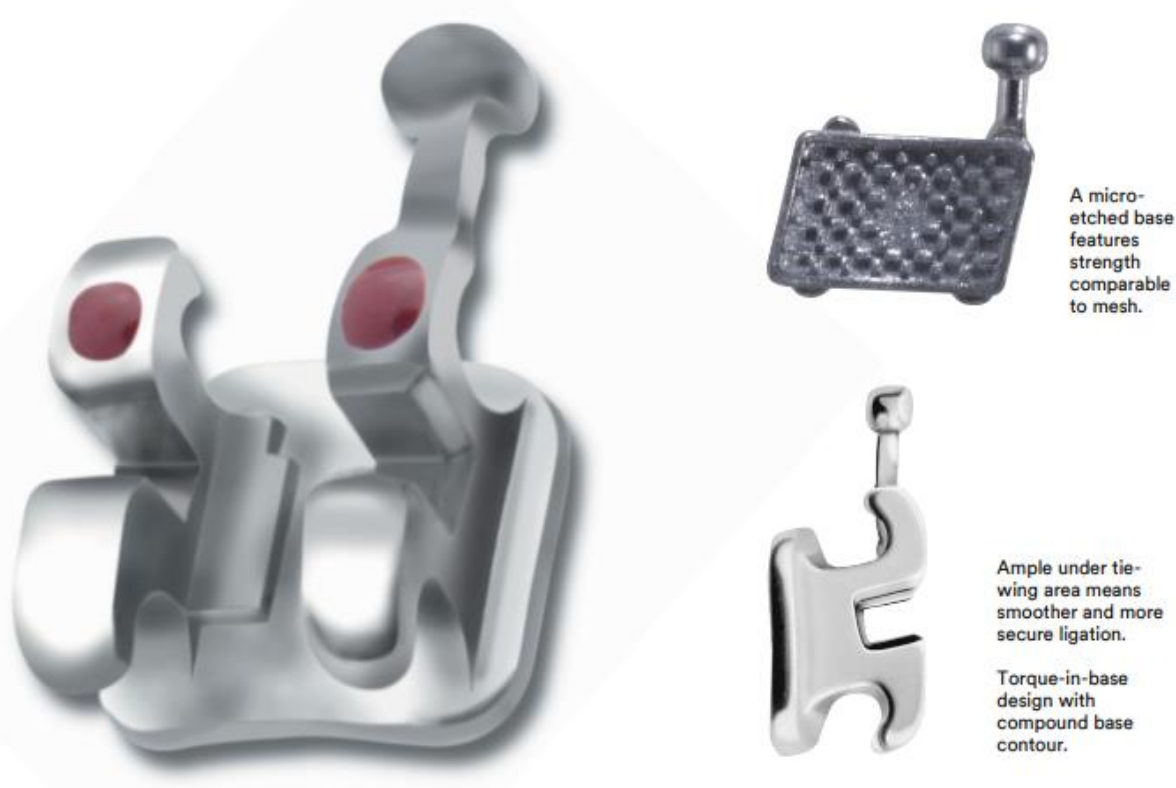

Figure 3: Miniature Twin (MIM, 17-4SS, micro-etched base)

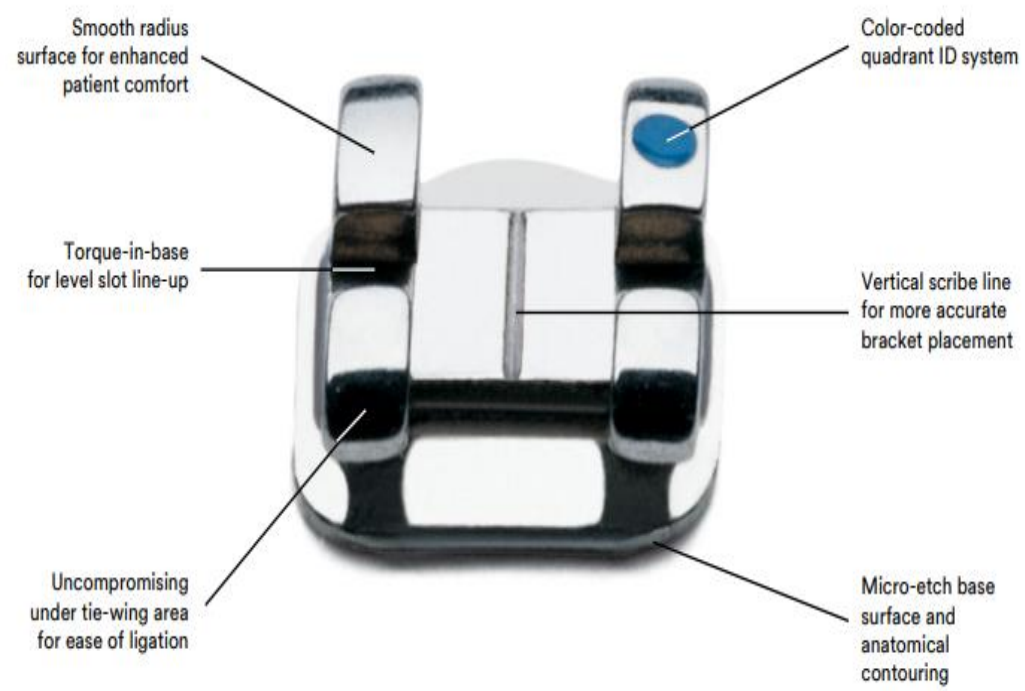

Figure 4: Victory Series LP (micro etch, 80 gauge mesh base) 


\section{American Orthodontics}

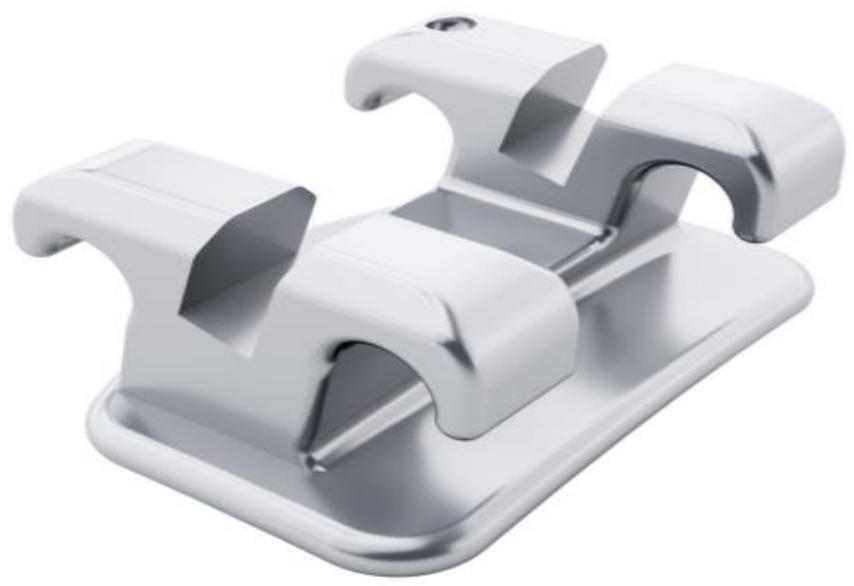

Figure 5: Master Series (maximum retention pads, 80 gauge mesh over etched foil base, photochemically etched pads)

\section{Forestadent}

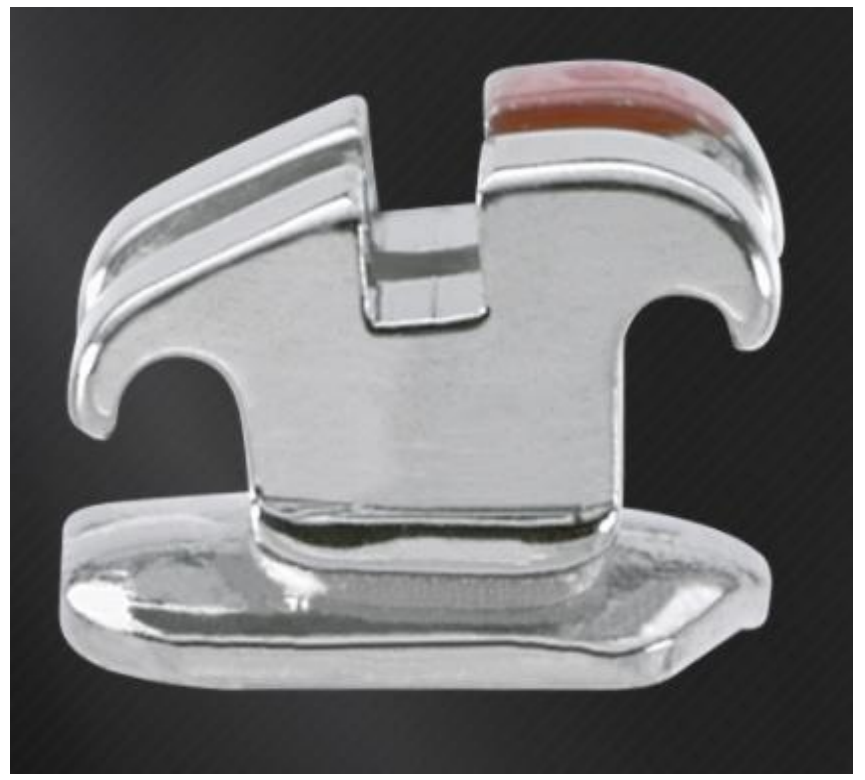

Figure 6: Mini-Mono (sandblasted mesh base) 


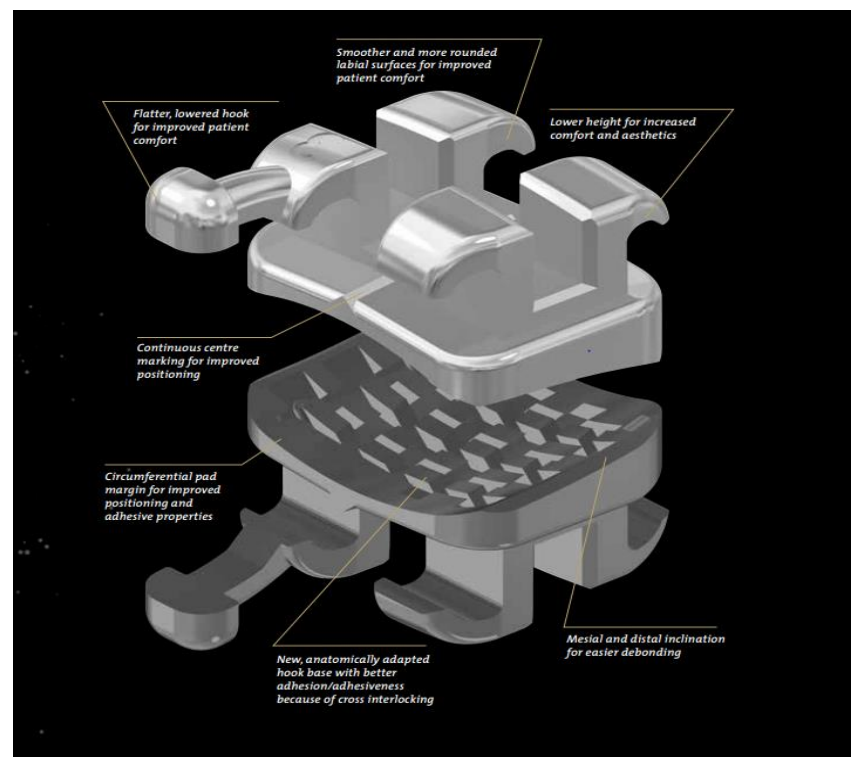

Figure 7: Mini Sprint II (MIM, medical nickel-free stainless steel)

\section{Rocky Mountain Orthodontics}

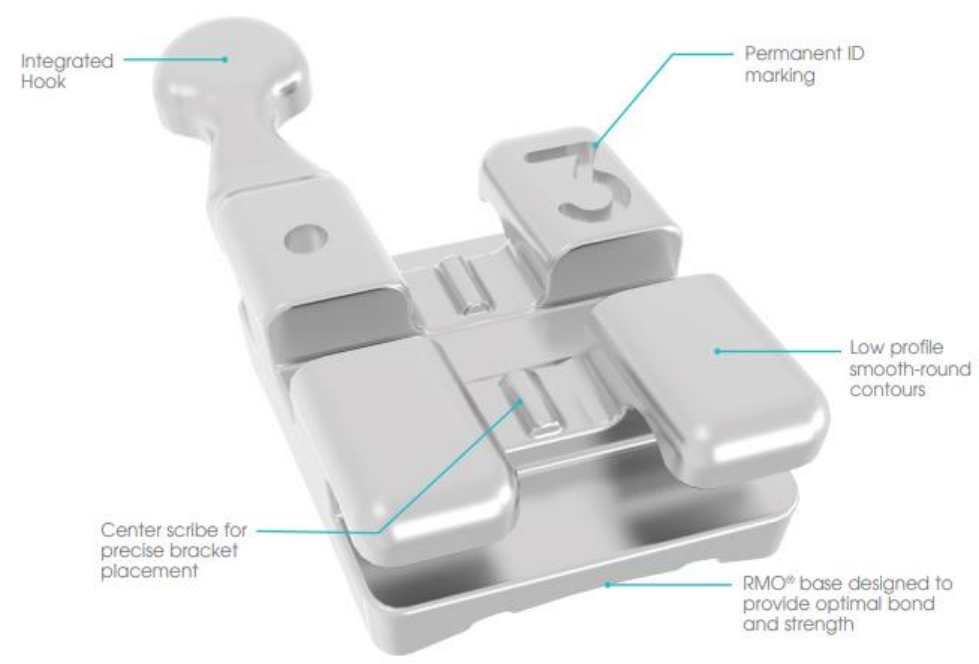

Figure 8: Integra (MIM, 316L stainless steel) 


\section{Dynaflex}

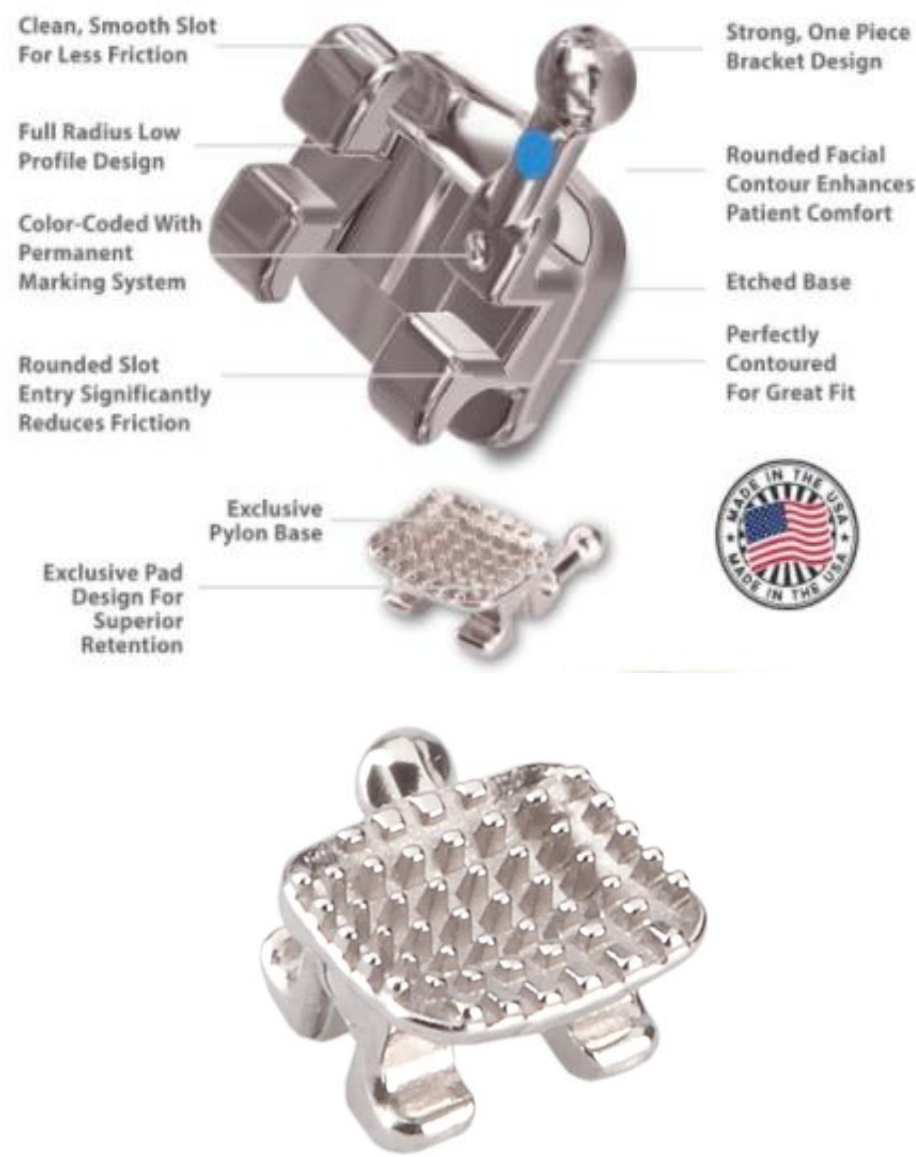

Figure 9: MTX (MIM, pylon base)

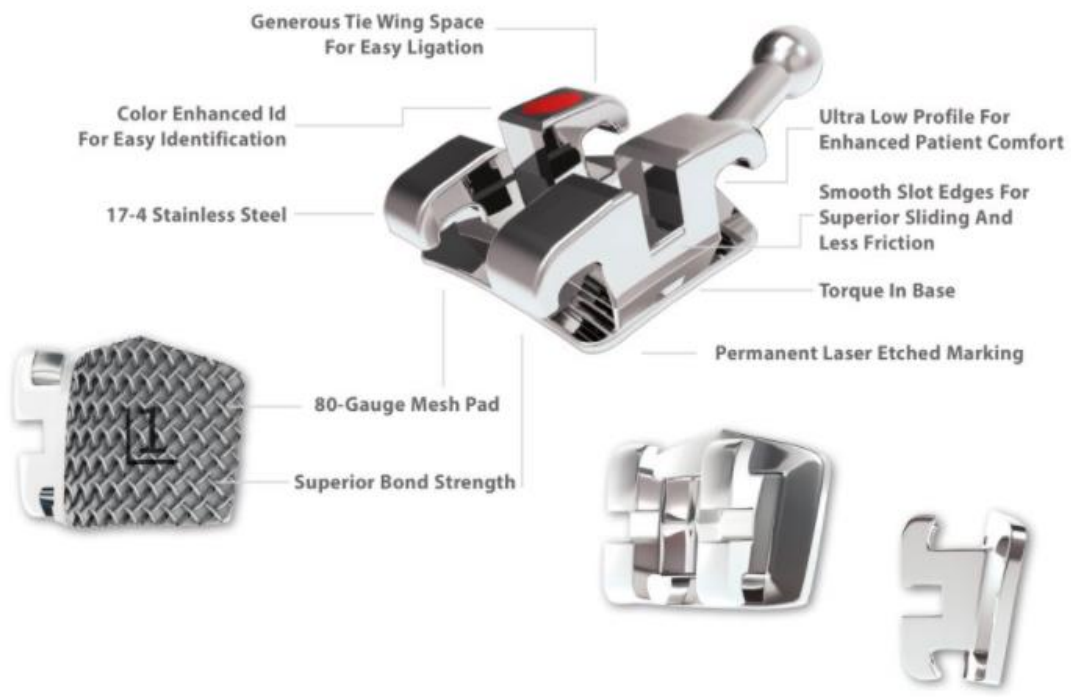

Figure 10: Atlas Mini (17-4SS, brazed 80 gauge foil mesh pad) 


\section{Dentaurum}

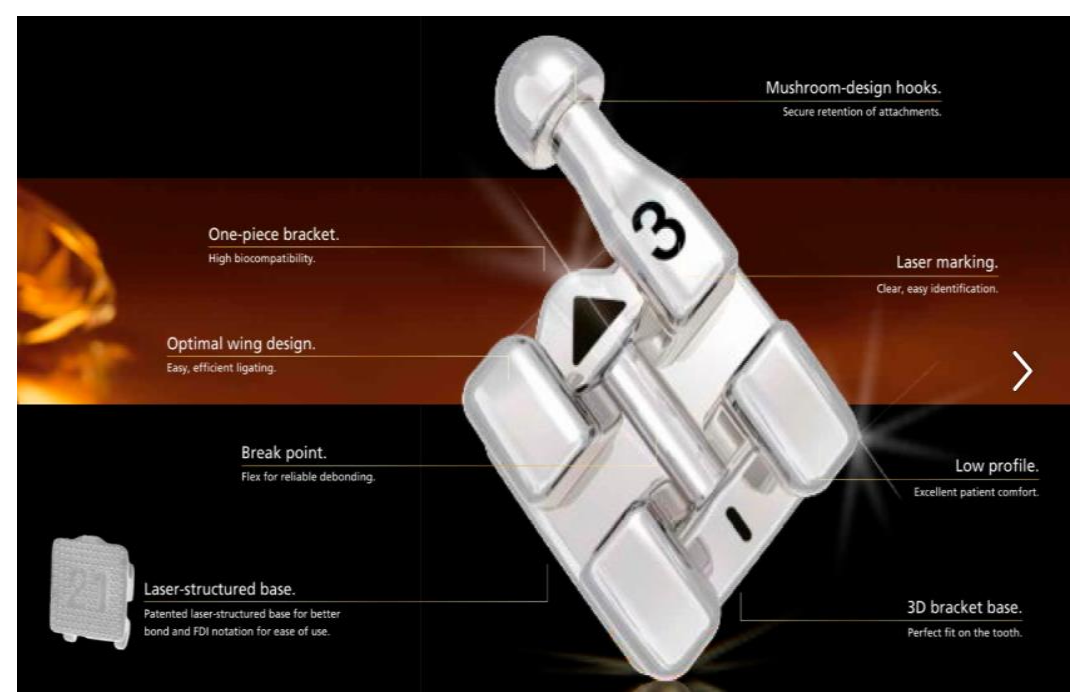

Figure 11: Discovery-Smart (MIM)

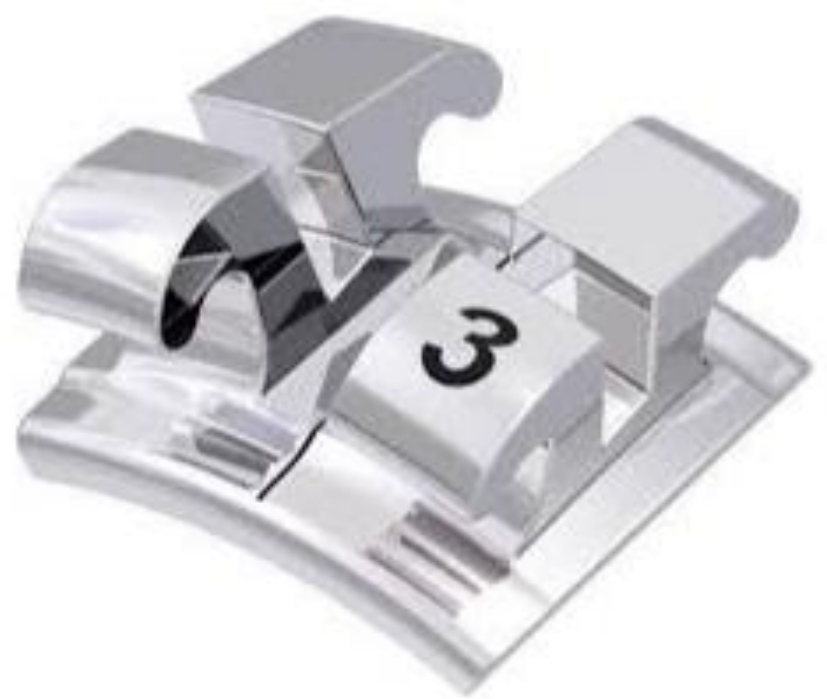

Figure 12: Equilibrium II 


\section{7. $\mathbf{G C}$}

\section{LEGEND medium}

The LEGENDTM collection is a traditional twin, metal bracket system available in a medium and mini size. LEGEND medium is manufactured by a Metal Injection Molding (MIM) and Computer Numerical Controlled (CNC) precision milling process for a consistent and precise bracket that features a $100 \%$ accurate slot and anatomically correct bonding pads for maximum bond retention.

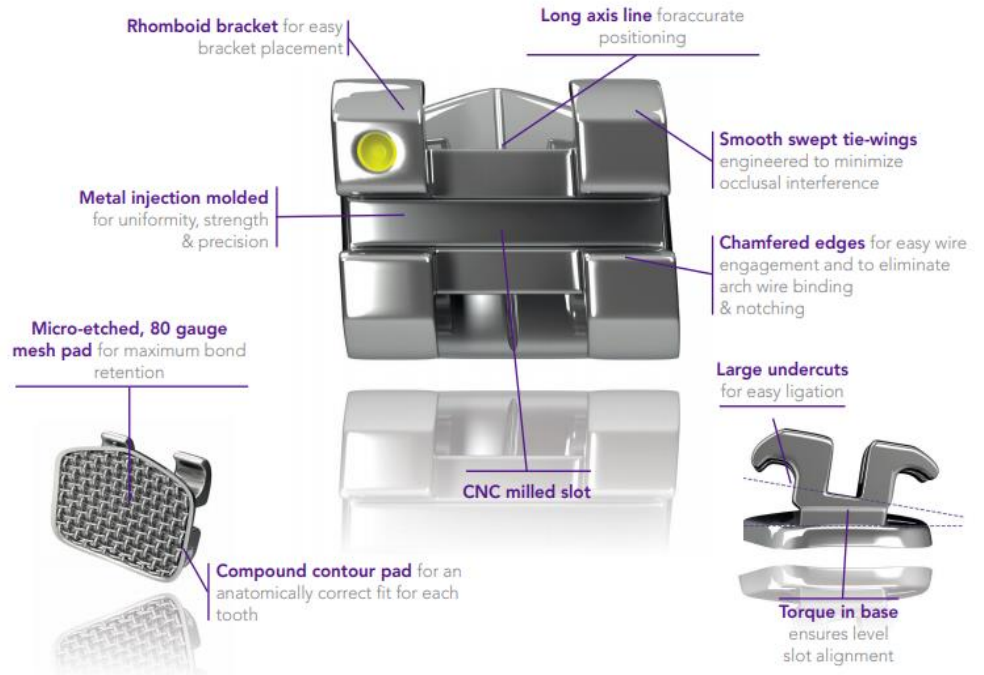

Figure 13: Legend medium bracket

\section{Ormco}

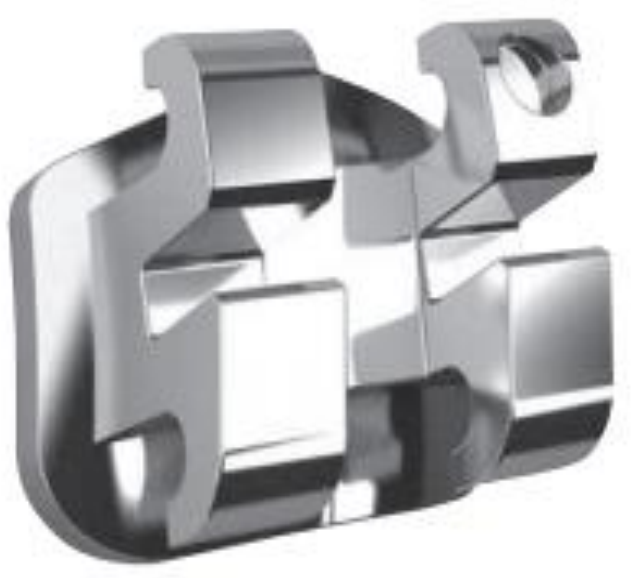

Figure 14: Mini Diamond Twin 


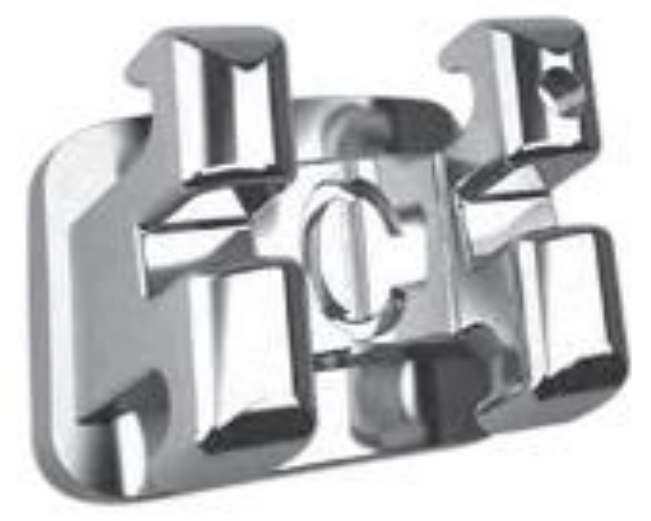

Figure 15: Mini-Twin

\begin{tabular}{|c|c|c|c|c|c|}
\hline Groups & Bracket name & $\begin{array}{l}\text { Manufacturing } \\
\text { method }\end{array}$ & Company & Pieces & Retention \\
\hline 1 & Miniature Twin & MIM & $3 \mathrm{M}$ & One-piece & Micro-etch, built-in \\
\hline$\overline{2}$ & Victory series & Machined (Milled) & $3 \mathrm{M}$ & Multiple pieces & $\begin{array}{l}\text { Micro-etch, added } 80 \text { gauge } \\
\text { mesh }\end{array}$ \\
\hline 3 & Master series & Machined (Milled) & American Ortho & Multiple pieces & $\begin{array}{l}\text { Photo-etch base, added } 80 \\
\text { gauge mesh }\end{array}$ \\
\hline$\overline{4}$ & Mini-Mono & Milled & Forestadent & Multiple pieces & Sandblasted base, added mesh \\
\hline 5 & MiniSprint II & MIM & Forestadent & One-piece & Hook base, built-in \\
\hline 6 & Integra & MIM & Rocky Mountain & One-piece & Built-in \\
\hline 7 & MTX & MIM & Dynaflex & One-piece & Pylon base, built-in \\
\hline 8 & Atlas Mini & Machined (Milled) & Dynaflex & Multiple pieces & Added 80 gauge mesh \\
\hline 9 & Discovery Smart & MIM & Dentaurum & One-piece & Laser structured built-in \\
\hline 10 & Equilibrium 2 & Milled & Dentaurum & One-piece & Laser structured built-in \\
\hline 11 & Legend Medium & MIM & GC & One-piece & $\begin{array}{l}\text { Micro etch, } 80 \text { gauge mesh built- } \\
\text { in }\end{array}$ \\
\hline 12 & Mini Diamond Twin & MIM & Ormco & Multiple pieces & 80 gauge mesh added \\
\hline 13 & Mini-Twin & MIM & Ormco & Multiple pieces & 80 gauge mesh added \\
\hline
\end{tabular}

Table 1: Bracket Summary

\section{Bonding Technique}

In vivo bonding of orthodontic brackets normally consists of several steps that were replicated in this study to most accurately represent typical clinical technique. Teeth are first isolated to maintain a dry working field necessary for ideal bonding after the etching is complete. The facial surfaces of teeth are etched with $37 \%$ phosphoric acid, rinsed and dried. Many orthodontists will 
then apply a bond material, such as Assure Plus ${ }^{\circledR}$, to the etched surface of enamel; this step was included in this study. The bond is lightly air dried and cured. Then the bracket is then pasted with composite, placed on the tooth, sighted, excess composite removed, and cured. In many studies, extracted teeth are used to bond brackets to test shear bond strength and assign an ARI score. However, using extracted teeth adds another degree of variation as all teeth are not created equally and usually healthy teeth are not extracted. For this study, we standardized the material to which the brackets would be bonded. Stainless steel tubes were used in place of extracted teeth. The tubes were prepared by Vince Kish and each was cut to a similar length; however, length was not relevant for the purpose of this study. Each tube had the same circumference and area of curvature. This was necessary to standardize the bonding surface for each bracket. Stainless steel tubes were sand-blasted in preparation for bonding. One uniform coat of Assure Plus ${ }^{\circledR}$ was applied to the tube surface, lightly dried and light cured for ten seconds. According to the manufacturer, Assure Plus ${ }^{\circledR}$ can be used under any chemical, light or dual cure adhesive, regardless of manufacturer. This bond can be used on wet or dry normal or atypical enamel, dentin, cementum, composite, gold, amalgam, stainless steel, zirconia, acrylic and porcelain. Assure Plus® consists of BisGMA, Ethanol, 2-Hydroxyethyl Methacrylate, and10Methacryloyloxydecyl Dihydrogen Phosphate. Tubes were loaded into the mounting jig and tightened in place with an adjustable screw. GoTo® bonding adhesive was then applied to the back of each bracket using a buttering technique to ensure adequate coverage and engagement of retentive components on bracket pad. Brackets were then placed on tube with equal pressure to allow composite expression on three sides of each bracket. The fourth side was placed in parallel to mounting jig top surface, where no composite expression was possible. This ensured dimension accuracy and perpendicular placement of brackets to debonding force of the Instron 
machine. Excess flash was removed. Brackets were light cured for ten seconds at each bracket edge. A total of 130 brackets were bonded to 39 tubes in groups of three based on bracket. Two tubes in each group of three had four brackets mounted $90^{\circ}$ apart from one another around the circumference of the tube. One tube in each group of three had two brackets mounted $180^{\circ}$ apart from one another around the circumference of the tube.

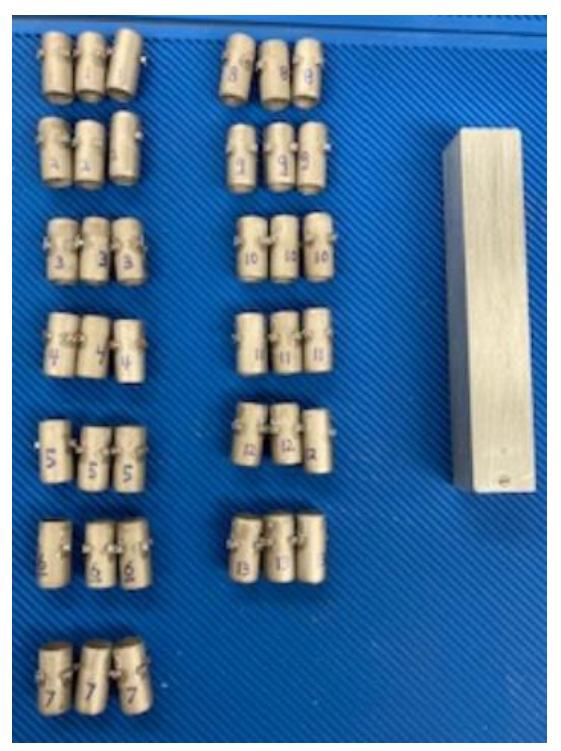

Figure 16: Brackets mounted to stainless steel tubes with mounting jig

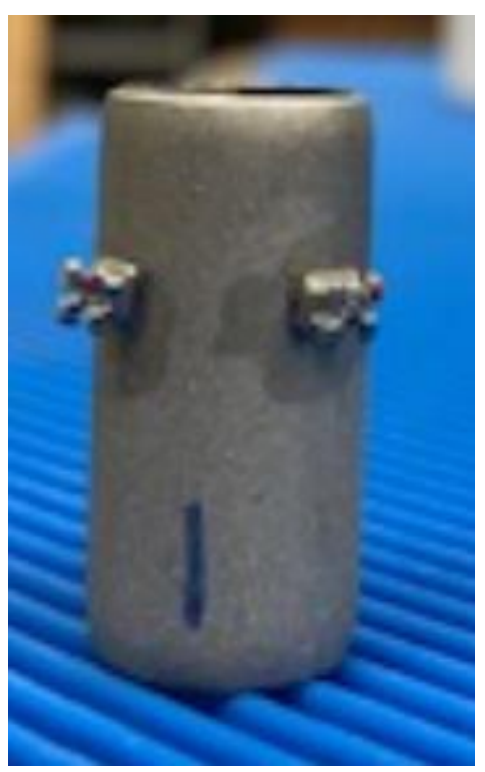

Figure 17: Brackets mounted to stainless steel tube 


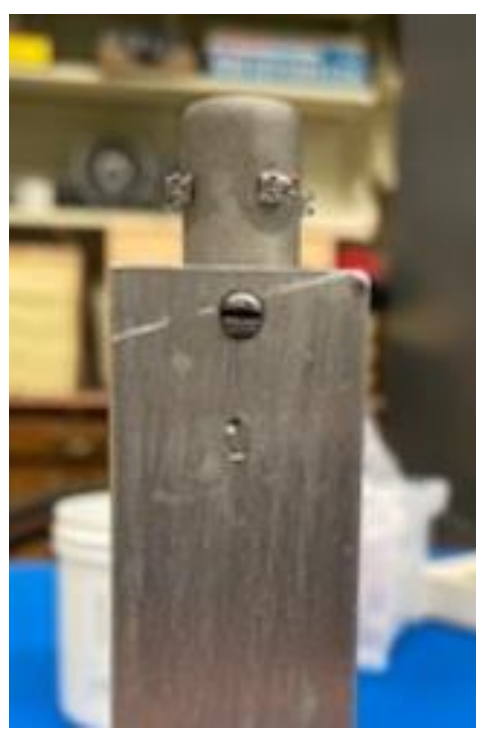

Figure 18: Stainless steel mounting tube in mounting jig with brackets previously bonded.

\section{Instron Machine}

All brackets were placed in a calibrated Instron machine and subjected to a force adequate for bracket debonding. Tubes with bonded brackets were placed in custom mounting jig and secured in place with a vice. Debonding apparatus consisted of 0.016 " stainless steel wire bent into loop shape and able to engage bracket under lower tie wings. The apparatus was designed to apply a debonding force occlusogingivally. The debonding apparatus was lowered to allow placement of the metal loop around the tie wings of each bracket. A nitrile glove fingertip was placed around bracket, tube and debonding apparatus to minimize bracket projectile motion on debonding. The Instron test was started and completed after the bracket was completely debonded from the tube surface. The vice was loosened, and tube rotated 90 or $180^{\circ}$ to align next bracket for testing. Vice was retightened and experiment repeated. Force of debond was given in Newtons and recorded by operator during experimental procedure. In a study by Lindemuth and Hagge to determine Instron crosshead speed necessary for bond failures, they found that crosshead speeds in testing range from $.05 \mathrm{~mm} / \mathrm{min}-50 \mathrm{~mm} / \mathrm{min}$. The viscoelasticity of adhesives is affected by the 
rate of stress application. Slower speeds allow for increased recovery, thus increased SBSs;

while faster speeds can induce brittle behavior, thus also increasing SBSs. The SBS and mode of failure was not statistically significant at various crosshead speeds. In a study comparing stainless steel orthodontic brackets with different adhesives, Sharma et al. found that SBS is the main factor needed in consideration of the success of bonding evolution. Brackets must be able to withstand intraoral forces during ortho treatment. Reynolds showed that 5.9-7.8 $\mathrm{MPa}$ resistances are sufficient ${ }^{17}$.

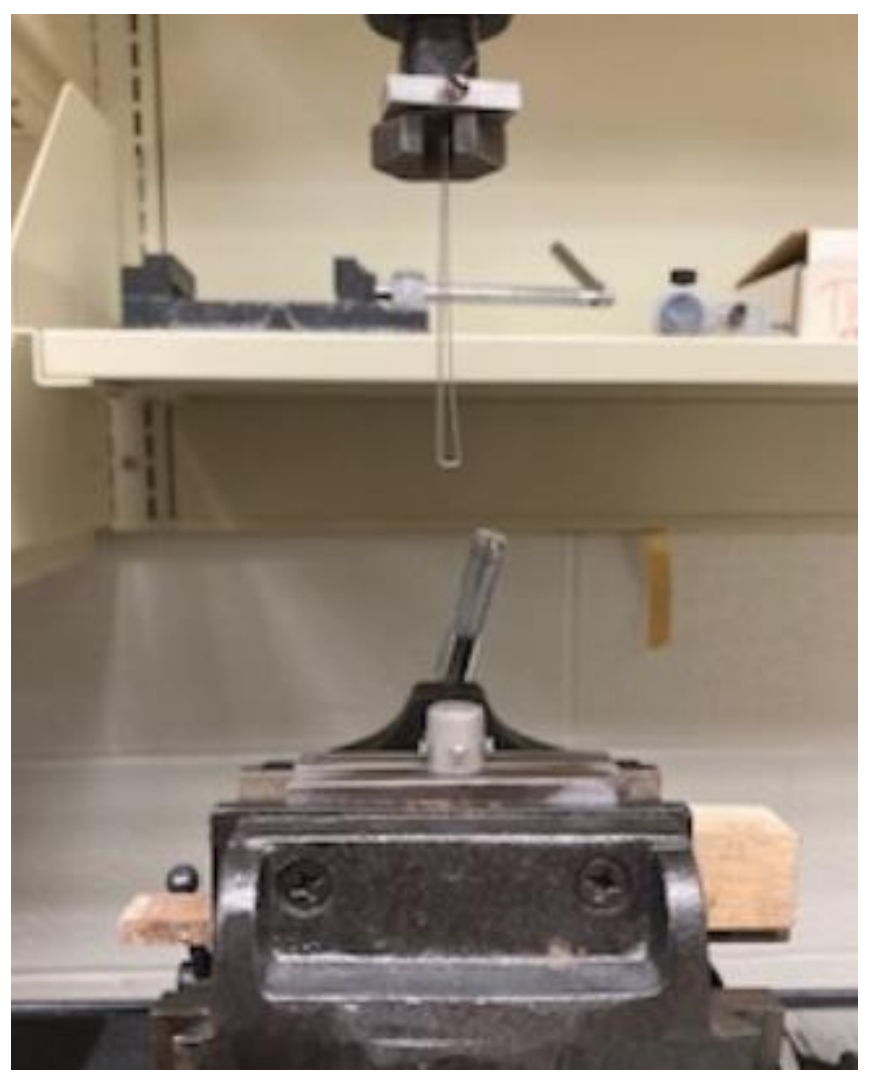

Figure 19: Stainless steel mounting tube with brackets bonded placed in Instron machine with debonding apparatus above. 


\section{After Debonding}

Once each bracket was debonded from respective tube, brackets were separated into individually labeled storage containers until ready for review. Brackets were observed by naked eye and with optical microscope at 10x power to determine Adhesive Remnant Index (ARI) score. A modified ARI score was used to score each bracket. This modified scale consisted of scores ranging from 0-5. A score of 0 indicates no adhesive left on the tube. A score of 1 indicates $1-25 \%$ of adhesive left on the tube. A score of 2 indicates $26-50 \%$ of adhesive left on tube. A score of 3 indicates $51-75 \%$ of adhesive left on tube. A score of 4 indicates $76-99 \%$ of adhesive left on tooth. Finally, a score of 5 indicates all adhesive left on the tube. All ARI scores were recorded at the time of observation. A second observation took place two weeks later to increase intraobserver accuracy. ARI scores were recorded and data sets were sent to statistician for evaluation.

\begin{tabular}{lr}
\hline Scores & Adhesive Remnant Index \\
\hline 0 & No adhesive remained on enamel \\
1 & Less than $50 \%$ of the adhesive remained on enamel \\
2 & More than $50 \%$ of the adhesive remained on enamel \\
3 & All adhesive remained on enamel
\end{tabular}

Table 2: Original ARI

\begin{tabular}{ll} 
Score & Definition \\
\hline 0 & No adhesive left on the tooth \\
1 & $1 \%-25 \%$ of adhesive left on the tooth \\
2 & $26 \%-50 \%$ of adhesive left on the tooth \\
3 & $51 \%-75 \%$ of adhesive left on the tooth \\
4 & $76 \%-99 \%$ of adhesive left on the tooth \\
5 & All adhesive left on the tooth with distinct \\
& impression of bracket mesh \\
\hline
\end{tabular}

Table 3: Modifed ARI 


\section{Statistical Analysis}

SAS (version 9.4, 2013, SAS Institute Inc. Cary, NC) was used to conduct the statistical analysis for the current study. Two sample $t$ test was incorporated to compare the difference in mean shear bonding strength and ARI scores between the two bracket designs, MIM brackets and Milled/cast brackets. Intra-class correlation coefficients (ICC) were calculated to evaluate the reliability of the measurements. We considered statistical significance for all tests with a twosided p-value of less than 0.05 .

\section{Chapter 4: Results}

A total of 130 brackets, 80 one-piece MIM and 50 multi-piece brackets, from top bracket manufacturing companies, were included in the current study. The shear bond strength of the brackets was calculated by dividing the debonding force by the pad area. The units of force in newtons $(\mathrm{N})$ were thus converted to megapascals $(\mathrm{MPa})$. The mean shear bonding strength and ARI scores for the study sample were $3.7 \pm 1.7$ and $4.2 \pm 0.7$ respectively. The results of two sample $t$ test showed that there was no significant difference between the one-piece MIM brackets $(3.5 \pm 1.7)$ and the multi-piece brackets $(4.0 \pm 1.8)$ in mean shear bonding strength $(\mathrm{t}(128)=1.33, \mathrm{p}=0.18)$. The mean of ARI scores for the multi-piece brackets $(4.4 \pm 0.7)$ is significantly higher than the mean of ARI scores for the MIM brackets $(4.0 \pm 0.7)(t(128)=3.5$, $\mathrm{p}=0.006$ ). For error measurements, all brackets' ARI scores were measured by the same researcher a second time with a two-week interval in between. The intra-class correlation coefficient $[\mathrm{ICC}=0.95,95 \% \mathrm{CL}(0.94-0.97)]$ indicates a high level of agreement between the two sets of ARI measurements. 


\section{Results Tables and Figures}

\begin{tabular}{|r|r|r|r|r|}
\hline \multicolumn{5}{|c|}{ ari score } \\
\hline ARI & Frequency & Percent & $\begin{array}{r}\text { Cumulative } \\
\text { Frequency }\end{array}$ & $\begin{array}{r}\text { Cumulative } \\
\text { Percent }\end{array}$ \\
\hline $\mathbf{2}$ & 2 & 1.54 & 2 & 1.54 \\
\hline $\mathbf{3}$ & 19 & 14.62 & 21 & 16.15 \\
\hline $\mathbf{4}$ & 64 & 49.23 & 85 & 65.38 \\
\hline $\mathbf{5}$ & 45 & 34.62 & 130 & 100.00 \\
\hline
\end{tabular}

Table 4: Distribution of ARI scores assigned to debonded brackets.

The UNIVARIATE Procedure

Variable: ARI (ari score)

\begin{tabular}{|l|r|l|r|}
\hline \multicolumn{4}{|c|}{ Moments } \\
\hline N & 50 & Sum Weights & 50 \\
\hline Mean & 4.44 & Sum Observations & 222 \\
\hline Std Deviation & 0.70450446 & Variance & 0.49632653 \\
\hline Skewness & -1.2379222 & Kurtosis & 1.6496126 \\
\hline Uncorrected SS & 1010 & Corrected SS & 24.32 \\
\hline Coeff Variation & 15.8672175 & Std Error Mean & 0.09963198 \\
\hline
\end{tabular}

\begin{tabular}{|l|l|l|l|}
\hline \multicolumn{4}{|c|}{ Basic Statistical Measures } \\
\hline \multicolumn{2}{|c|}{ Location } & \multicolumn{2}{c|}{ Variability } \\
\hline Mean & 4.440000 & Std Deviation & 0.70450 \\
\hline Median & 5.000000 & Variance & 0.49633 \\
\hline Mode & 5.000000 & Range & 3.00000 \\
\hline & & Interquartile Range & 1.00000 \\
\hline
\end{tabular}




\begin{tabular}{|r|r|r|r|}
\hline \multicolumn{3}{|c|}{ Extreme Observations } \\
\hline \multicolumn{2}{|c|}{ Lowest } & \multicolumn{2}{c|}{ Highest } \\
\hline Value & Obs & Value & Obs \\
\hline 2 & 7 & 5 & 35 \\
\hline 3 & 46 & 5 & 36 \\
\hline 3 & 9 & 5 & 37 \\
\hline 3 & 8 & 5 & 39 \\
\hline 4 & 50 & 5 & 40 \\
\hline
\end{tabular}

Table 5 a,b,c: Moments (a), basic measurements (b) and extreme observations (c) for ARI scores of multi-piece brackets

The UNIVARIATE Procedure Variable: sbs (shear bonding strength=debonding force/pad area)

\begin{tabular}{|l|r|l|r|}
\hline \multicolumn{4}{|c|}{ Moments } \\
\hline N & 50 & Sum Weights & 50 \\
\hline Mean & 3.9642 & Sum Observations & 198.21 \\
\hline Std Deviation & 1.82669333 & Variance & 3.33680853 \\
\hline Skewness & 0.65925974 & Kurtosis & 0.57650459 \\
\hline Uncorrected SS & 949.2477 & Corrected SS & 163.503618 \\
\hline Coeff Variation & 46.079747 & Std Error Mean & 0.25833345 \\
\hline
\end{tabular}

\begin{tabular}{|l|l|l|l|}
\hline \multicolumn{4}{|c|}{ Basic Statistical Measures } \\
\hline \multicolumn{2}{|c|}{ Location } & \multicolumn{2}{c|}{ Variability } \\
\hline Mean & 3.964200 & Std Deviation & 1.82669 \\
\hline Median & 3.860000 & Variance & 3.33681 \\
\hline Mode & 1.690000 & Range & 8.93000 \\
\hline & & Interquartile Range & 2.26000 \\
\hline
\end{tabular}




\begin{tabular}{|r|r|r|r|}
\hline \multicolumn{3}{|c|}{ Extreme Observations } \\
\hline \multicolumn{2}{|c|}{ Lowest } & \multicolumn{2}{c|}{ Highest } \\
\hline Value & Obs & Value & Obs \\
\hline 0.57 & 24 & 6.25 & 17 \\
\hline 1.54 & 40 & 7.19 & 12 \\
\hline 1.67 & 34 & 7.54 & 7 \\
\hline 1.69 & 31 & 7.56 & 20 \\
\hline 1.69 & 30 & 9.50 & 46 \\
\hline
\end{tabular}

Table 6 a,b,c: Moments (a), basic measurements (b) and extreme observations (c) for shear bond strength of multi-piece brackets.

\section{The UNIVARIATE Procedure}

Variable: ARI (ari score)

\begin{tabular}{|l|r|l|r|}
\hline \multicolumn{3}{|c|}{ Moments } \\
\hline N & 80 & Sum Weights & 80 \\
\hline Mean & 4 & Sum Observations & 320 \\
\hline Std Deviation & 0.69355076 & Variance & 0.48101266 \\
\hline Skewness & -0.2334989 & Kurtosis & -0.1660403 \\
\hline Uncorrected SS & 1318 & Corrected SS & 38 \\
\hline Coeff Variation & 17.338769 & Std Error Mean & 0.07754133 \\
\hline
\end{tabular}

\begin{tabular}{|l|l|l|r|}
\hline \multicolumn{4}{|c|}{ Basic Statistical Measures } \\
\hline \multicolumn{2}{|c|}{ Location } & \multicolumn{2}{c|}{ Variability } \\
\hline Mean & 4.000000 & Std Deviation & 0.69355 \\
\hline Median & 4.000000 & Variance & 0.48101 \\
\hline Mode & 4.000000 & Range & 3.00000 \\
\hline & & Interquartile Range & 0 \\
\hline
\end{tabular}




\begin{tabular}{|r|r|r|r|}
\hline \multicolumn{3}{|c|}{ Extreme Observations } \\
\hline \multicolumn{2}{|c|}{ Lowest } & \multicolumn{2}{c|}{ Highest } \\
\hline Value & Obs & Value & Obs \\
\hline 2 & 110 & 5 & 112 \\
\hline 3 & 129 & 5 & 113 \\
\hline 3 & 115 & 5 & 114 \\
\hline 3 & 109 & 5 & 125 \\
\hline 3 & 100 & 5 & 128 \\
\hline
\end{tabular}

Table 7 a,b,c: Moments (a), basic measurements (b) and extreme observations (c) for ARI scores of one-piece. Brackets

The UNIVARIATE Procedure Variable: sbs (shear bonding strength=debonding force/pad area)

\begin{tabular}{|l|r|l|r|}
\hline \multicolumn{4}{|c|}{ Moments } \\
\hline N & 80 & Sum Weights & 80 \\
\hline Mean & 3.5495 & Sum Observations & 283.96 \\
\hline Std Deviation & 1.65585108 & Variance & 2.74184278 \\
\hline Skewness & 1.02147716 & Kurtosis & 1.10570366 \\
\hline Uncorrected SS & 1224.5216 & Corrected SS & 216.60558 \\
\hline Coeff Variation & 46.6502627 & Std Error Mean & 0.18512978 \\
\hline
\end{tabular}

\begin{tabular}{|l|l|l|l|}
\hline \multicolumn{4}{|c|}{ Basic Statistical Measures } \\
\hline \multicolumn{2}{|c|}{ Location } & \multicolumn{2}{c|}{ Variability } \\
\hline Mean & 3.549500 & Std Deviation & 1.65585 \\
\hline Median & 3.275000 & Variance & 2.74184 \\
\hline Mode & 1.960000 & Range & 7.74000 \\
\hline & & Interquartile Range & 1.95500 \\
\hline
\end{tabular}




\begin{tabular}{|r|r|r|r|}
\hline \multicolumn{3}{|c|}{ Extreme Observations } \\
\hline \multicolumn{2}{|c|}{ Lowest } & \multicolumn{2}{c|}{ Highest } \\
\hline Value & Obs & Value & Obs \\
\hline 1.13 & 58 & 6.82 & 110 \\
\hline 1.16 & 67 & 6.84 & 96 \\
\hline 1.18 & 53 & 7.71 & 87 \\
\hline 1.23 & 122 & 8.07 & 99 \\
\hline 1.31 & 69 & 8.87 & 93 \\
\hline
\end{tabular}

Table 8 a,b,c: Moments (a), basic measurements (b) and extreme observations (c) for shear bond strength of multi-piece brackets. 


\section{The TTEST Procedure}

Variable: sbs (shear bonding strength=debonding force/pad area)

\begin{tabular}{|l|r|r|r|r|r|r|}
\hline group & $\mathbf{N}$ & Mean & Std Dev & Std Err & Minimum & Maximum \\
\hline Milled & 50 & 3.9642 & 1.8267 & 0.2583 & 0.5700 & 9.5000 \\
\hline MIM & 80 & 3.5495 & 1.6559 & 0.1851 & 1.1300 & 8.8700 \\
\hline Diff (1-2) & & 0.4147 & 1.7233 & 0.3107 & & \\
\hline
\end{tabular}

\begin{tabular}{|l|l|r|r|r|r|r|l|}
\hline & \multirow{2}{*}{ group } & Method & Mean & \multicolumn{2}{|c|}{$95 \%$ CL Mean } & Std Dev & \multicolumn{2}{|c|}{$\begin{array}{c}95 \% \\
\text { CL Std Dev }\end{array}$} \\
\hline Milled & & 3.9642 & 3.4451 & 4.4833 & 1.8267 & 1.5259 & 2.2763 \\
\hline MIM & & 3.5495 & 3.1810 & 3.9180 & 1.6559 & 1.4331 & 1.9613 \\
\hline Diff (1-2) & Pooled & 0.4147 & -0.2000 & 1.0294 & 1.7233 & 1.5355 & 1.9637 \\
\hline Diff (1-2) & Satterthwaite & 0.4147 & -0.2161 & 1.0455 & & & \\
\hline
\end{tabular}

\begin{tabular}{|l|l|r|r|r|}
\hline Method & Variances & DF & $\mathbf{t}$ Value & $\operatorname{Pr}>|\mathbf{t}|$ \\
\hline Pooled & Equal & 128 & 1.33 & 0.1843 \\
\hline Satterthwaite & Unequal & 96.471 & 1.30 & 0.1951 \\
\hline
\end{tabular}

\begin{tabular}{|l|r|r|r|r|}
\hline \multicolumn{5}{|c|}{ Equality of Variances } \\
\hline Method & Num DF & Den DF & F Value & Pr > F \\
\hline Folded F & 49 & 79 & 1.22 & 0.4319 \\
\hline
\end{tabular}

Table 9 a,b,c,d: T-test values for measurements (a), differences (b), variances (c) and equality of variances $(d)$ of shear bond strength for one-piece and multi-piece brackets. 


\section{The TTEST Procedure}

Variable: sbs (shear bonding strength=debonding force/pad area)

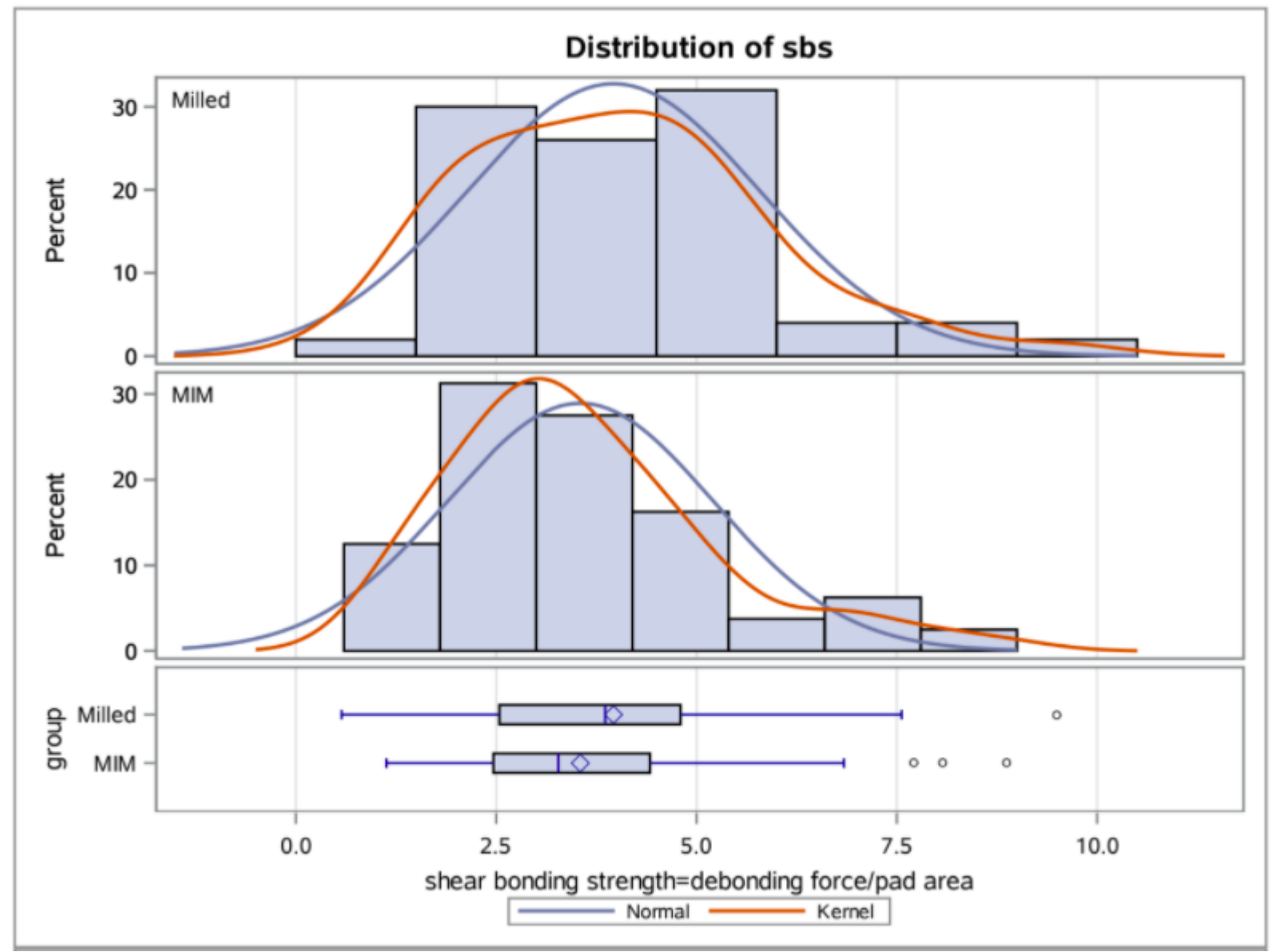

Figure 20: T-test distribution of shear bond strength of one-piece and multi-piece brackets. 


\section{The TTEST Procedure}

Variable: ARI (ari score)

Variable: ARI (ari score)

\begin{tabular}{|l|r|r|r|r|r|r|}
\hline group & $\mathbf{N}$ & Mean & Std Dev & Std Err & Minimum & Maximum \\
\hline Milled & 50 & 4.4400 & 0.7045 & 0.0996 & 2.0000 & 5.0000 \\
\hline MIM & 80 & 4.0000 & 0.6936 & 0.0775 & 2.0000 & 5.0000 \\
\hline Diff (1-2) & & 0.4400 & 0.6978 & 0.1258 & & \\
\hline
\end{tabular}

\begin{tabular}{|l|l|r|r|r|r|r|l|}
\hline & & & \multicolumn{2}{|c|}{} & \multicolumn{2}{|c|}{$95 \%$} \\
group & Method & Mean & \multicolumn{2}{|c|}{$95 \%$ CL Mean } & Std Dev & \multicolumn{2}{|c|}{ CL Std Dev } \\
\hline Milled & & 4.4400 & 4.2398 & 4.6402 & 0.7045 & 0.5885 & 0.8779 \\
\hline MIM & & 4.0000 & 3.8457 & 4.1543 & 0.6936 & 0.6002 & 0.8215 \\
\hline Diff (1-2) & Pooled & 0.4400 & 0.1911 & 0.6889 & 0.6978 & 0.6218 & 0.7951 \\
\hline Diff (1-2) & Satterthwaite & 0.4400 & 0.1896 & 0.6904 & & & \\
\hline
\end{tabular}

\begin{tabular}{|l|l|r|r|r|}
\hline Method & Variances & DF & $\mathbf{t}$ Value & $\operatorname{Pr}>|\mathbf{t}|$ \\
\hline Pooled & Equal & 128 & 3.50 & 0.0006 \\
\hline Satterthwaite & Unequal & 102.92 & 3.49 & 0.0007 \\
\hline
\end{tabular}

\begin{tabular}{|l|r|r|r|r|}
\hline \multicolumn{5}{|c|}{ Equality of Variances } \\
\hline Method & Num DF & Den DF & F Value & Pr > F \\
\hline Folded F & 49 & 79 & 1.03 & 0.8873 \\
\hline
\end{tabular}

Table 10 a,b,c,d: T-test values for measurements (a), differences (b), variances (c) and equality of variances (d) of ARI scores for one-piece and multi-piece brackets. 
The TTEST Procedure

Variable: ARI (ari score)

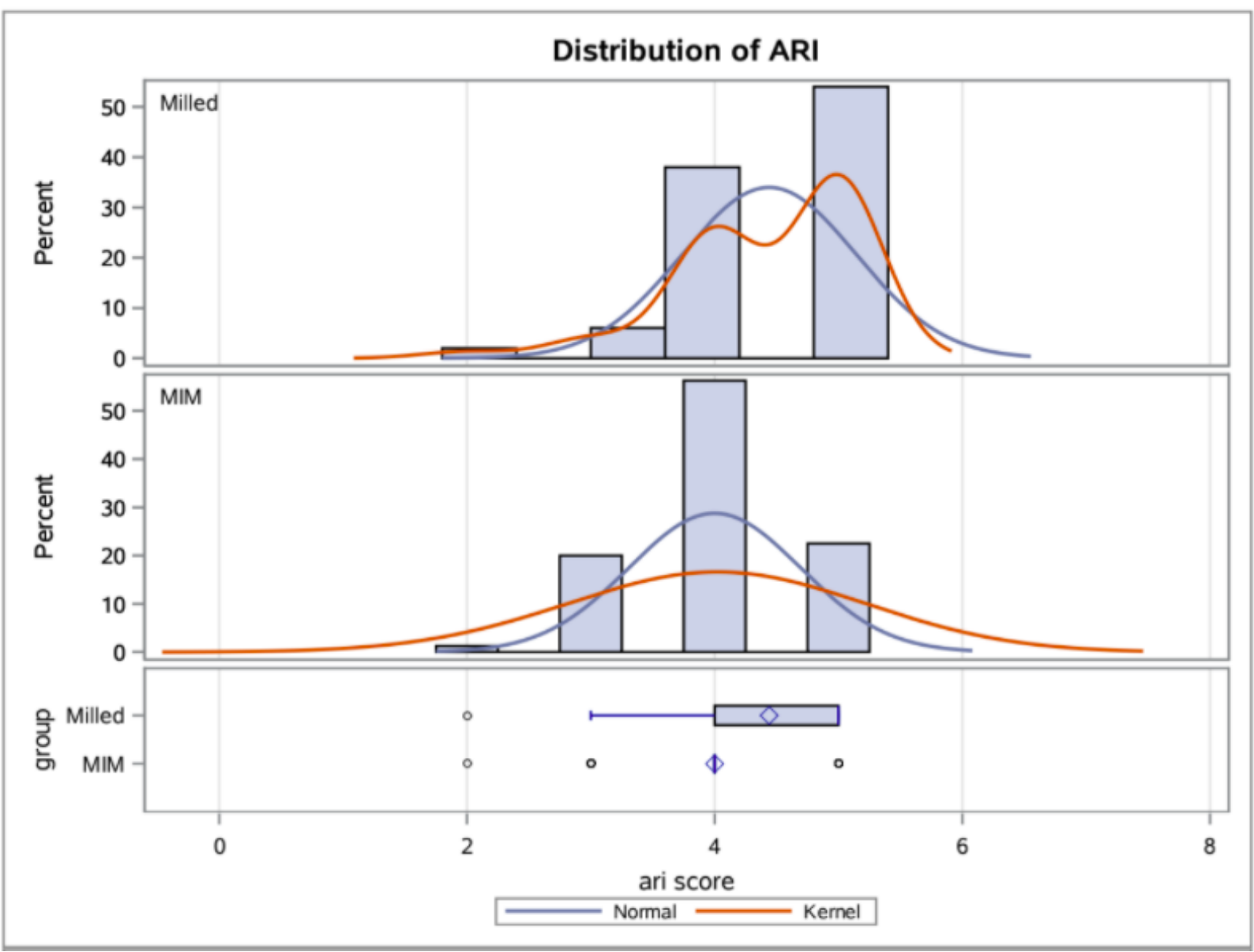

Figure 21: T-test distribution of ARI scores of one-piece and multi-piece brackets. 


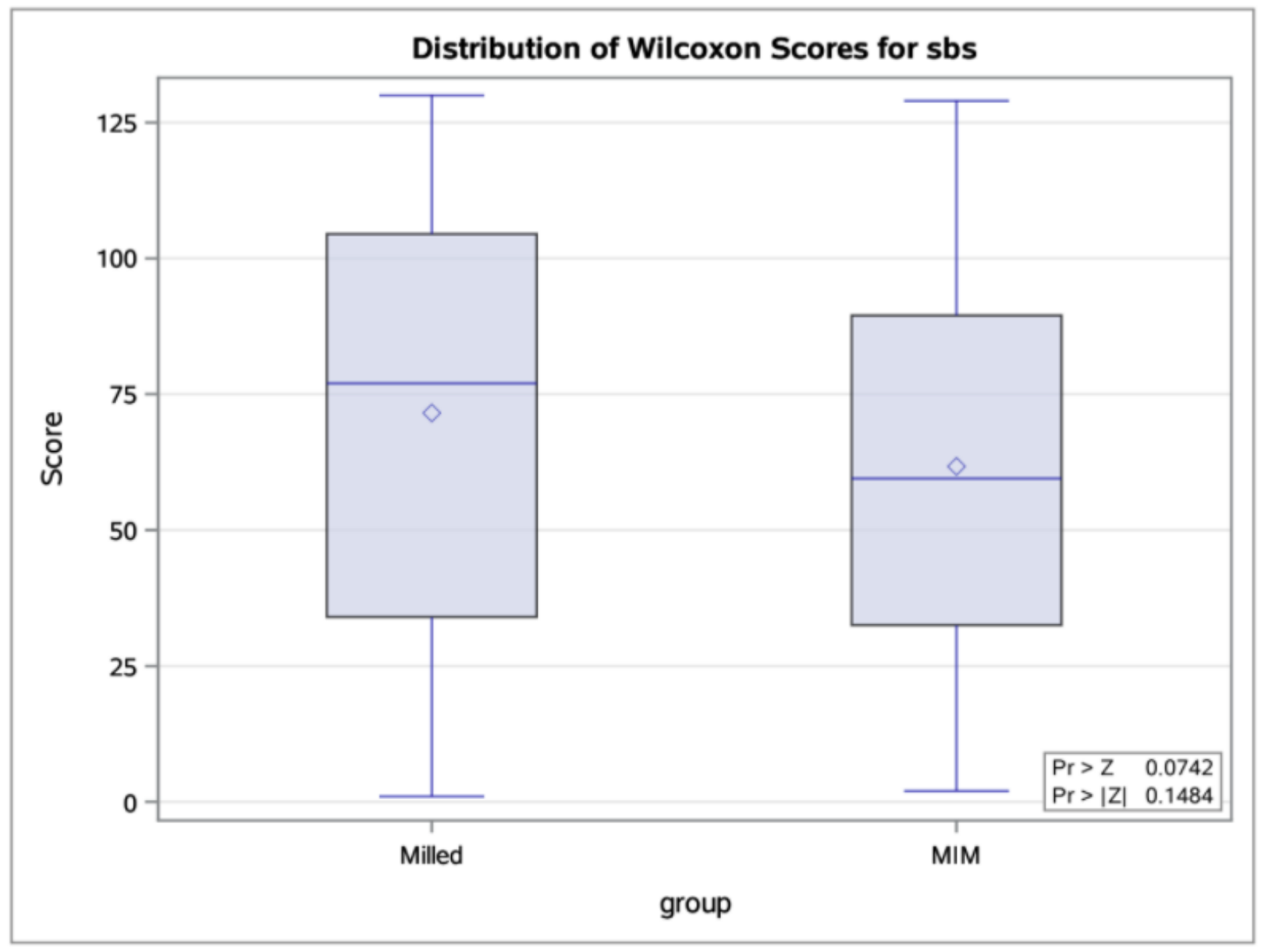

Figure 22: Wilcoxon distribution of shear bond strength of one-piece and multi-piece brackets. 


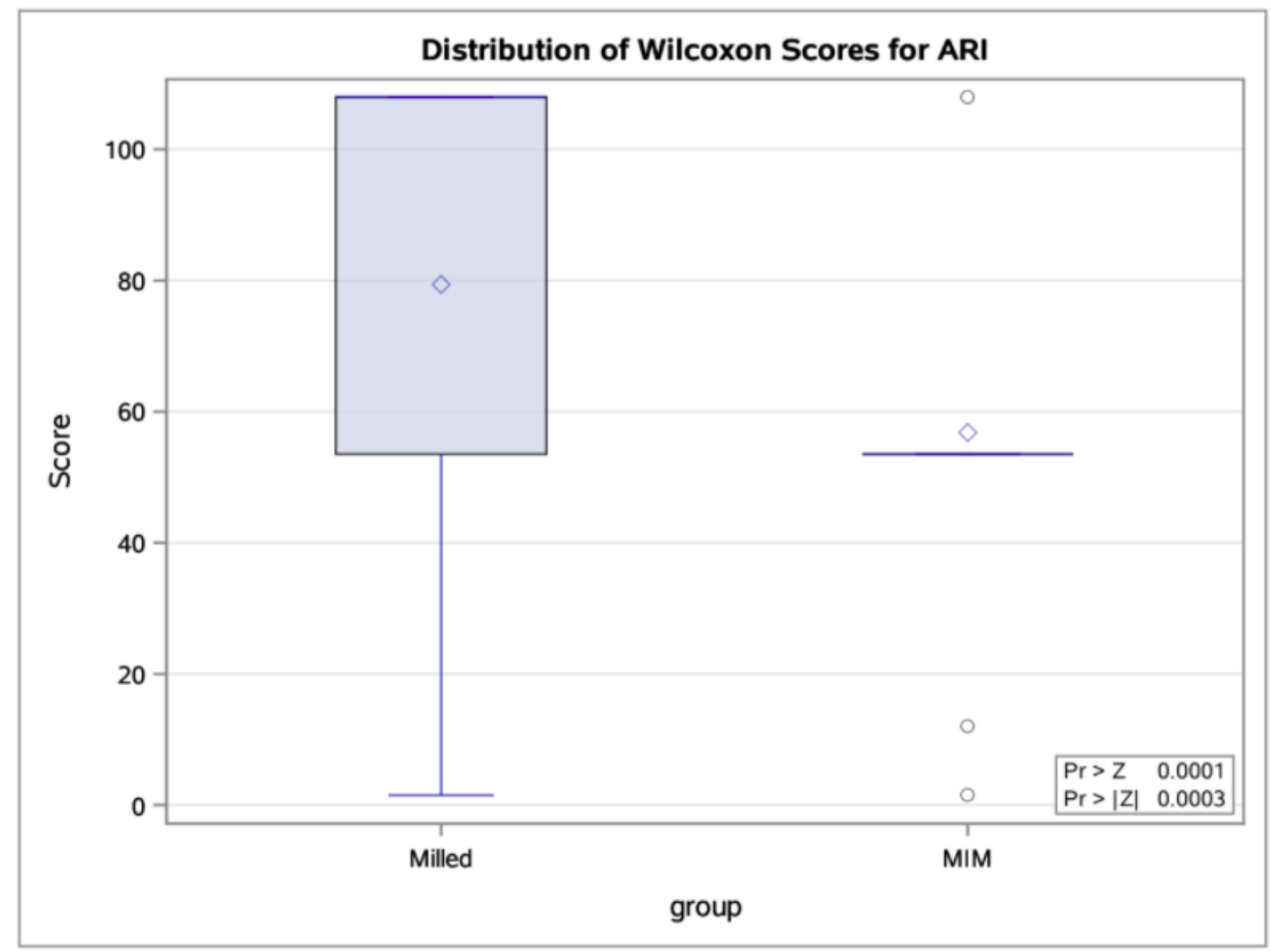

Figure 23: Wilcoxon distribution of ARI scores of one-piece and multi-piece brackets.

\section{Null Hypotheses Testing}

1. Accepted. There is no significant intergroup difference in shear bond strength between one-piece brackets manufactured by the MIM process and multi-piece brackets with components manufactured by either MIM, milling or casting process

2. Accepted. There is no significant intragroup difference in shear bond strength between different multi-piece brackets with components manufactured by MIM, milling, or casting. 
3. Accepted. There is no significant intragroup difference in shear bond strength between different one-piece brackets made by MIM.

4. Accepted. There is no significant intergroup difference in shear bond strength between one-piece or two-piece brackets manufactured by MIM, casting, or milling and bracket pad design/retentive features (ex. Micro-etching, photo-etching, addition of mesh, etc.)

5. Accepted. There is no significant intragroup difference in shear bond strength between one-piece brackets made by MIM and bracket pad design/retentive features.

6. Accepted. There is no significant intragroup difference in shear bond strength between multi-piece brackets made with components manufactured by MIM, milling, or casting and bracket pad design/retentive features.

7. Rejected. There is no significant difference in ARI score between one-piece MIM metal brackets and multi-piece brackets with components manufactured by MIM, milling, or casting.

\section{Chapter 5: Discussion}

\section{Clinical Implications:}

Emergency appointments in an orthodontic practice increase time and expense to both patient and provider. In many cases, these "emergencies" are 100\% preventable. Bracket failures are among the most common types of unscheduled visit ${ }^{1}$. They can be a source of frustration for both parties and may lead to a less pleasant overall treatment experience. If these failures become a common occurrence, treatment outcomes have an increased likelihood of suffering. Selecting a bracket that decreases the chance of bond failure would benefit both patient and provider. When brackets debond from the tooth surface, the failure can occur at one of three locations; the tooth- 
composite interface, the bracket-composite interface or within the composite itself. If the failure occurs at the tooth-composite interface, many times this is due to contamination of the prepared tooth surface at the time of bracket placement. If the surface of the tooth is contaminated after the etching procedure, surface area for composite acceptance by enamel can decrease significantly. If the failure occurs within the composite itself, this may due to a failure within the bonding system; more likely a manufacturing error than operator error as long as bonding instructions were followed as given. If the bond failure occurs at the bracket-composite interface, this is where selecting the right bracket with the right pad design becomes crucial. Bond failures here typically result from one of two scenarios; either the composite was inadequately applied or the bracket pad design is not sufficient to retain composite when subjected to force. In the case of application, the operator may not apply enough composite material to fill the entire surface area of the pad. To avoid this, the operator should ensure composite expression from all four sides of the bracket on placement. Additionally, the operator should make sure the depth of composite placement within the bracket pad is adequate to cover all available surface area. This may be accomplished by applying the composite in a buttering fashion, back-and-forth across the pad of the bracket. If composite application and amount are not the issue, then bracket selection can play a critical role. Choosing a bracket with the ideal amount of surface area to accept the most composite and minimize the likelihood of breakage is imperative to successful outcomes in practice. That being said, many other variables in practice need to be considered and ultimately a provider should choose what bracket works best within their practice. Other factors to be considered before buying a particular bracket would be bonding system used, staff preference, ease of use, patient comfort and esthetics to name a few. Brackets with larger bases and thus greater pad surface area will tend to show better results in debond studies ${ }^{12-14}$. This is obviously 
due to the increased surface area for composite acceptance. However, as the size of the bracket increases, patient comfort decreases. Larger brackets may be more difficult to handle and harder to place for some providers. Other providers will have greater difficultly in handling and placement of smaller brackets. Again, providers should select what works best for them in their respective practice as well as wat will lead them to the best treatment outcomes. Not all brackets are created equally, as such, neither are orthodontists. The results of this study indicate that there is no difference in one-piece MIM brackets and multi-piece brackets in shear bond strength.

However, ARI scores were significantly different. Multi-piece brackets had significantly higher ARI scores, meaning more composite was left on the stainless steel tube when compared with the amount left on the tube by one-piece MIM brackets. Thus, within the brackets studied, one-piece MIM brackets on average retained composite better than multi-piece brackets. The majority of multi-piece brackets had an added layer of 80 gauge mesh brazed onto the base, while one-piece MIM brackets had built-in retentive features such as pylons. Operator error could have contributed to this finding; further research is needed.

\section{Recommendations:}

Further in-vitro studies are needed to verify the accuracy of this study's results. The variety of brackets on the market is vast and with so many choices, any provider can easily become overwhelmed when deciding which product is right for them. Testing brackets form different companies than the ones used in this study would be beneficial for a source of more data on the

topic. Testing brackets made the same company but manufactured by different methods may also prove useful in eliminating variation in between different companies. MIM processes may have slight variations between different companies that could lead to differences in clinical results. Machining brackets could also be done by several different methods by various companies and 
even within a single company. Testing multi-piece brackets that are manufactured the same way would eliminate an additional variable from this study. For example, selecting multi-piece brackets that are soldered together instead of brazed could give additional data worth investigating. Testing brackets for different teeth could provide interesting results as well. In this study, we observed upper lateral incisor brackets only. In future studies, testing central incisor, canine, premolar or molar brackets could lead to different conclusions. This may be due to the different forces experienced by each bracket depending on the location within the mouth. Incisor brackets will experience a shear force, while canine brackets will feel a ripping/tearing force. Molar and premolar brackets will experience a grinding force and may be more likely to fail due to the increased usage of posterior teeth during the chewing cycle. This study used a single operator to bond all brackets in order to decrease inconsistencies between multiple operators. In order to better calibrate a future operator, multiple operators should be used to initially to bond sample brackets and test each to ensure consistent debonding forces. This will allow the study operator to know the range of force (newtons) within which each bracket should fail. The bonding technique recommended by the bond manufacturer was followed for the specific surface being bonded, in this case stainless steel, in this study and would be recommended for future studies. Different bonding systems may be utilized as long as all brackets within the study are subjected to the same system and techniques.

\section{Chapter 6: Summary and Conclusion}

The purpose of the study was to determine if there is a significant difference in durability, shear bond strength and debonding characteristics between one-piece metal brackets made by metal injection molding and multi-piece metal brackets made by machining then soldering/brazing 
with differing pad designs. Upon contacting top orthodontic bracket manufacturers, 130 different multi- and one-piece metal orthodontic brackets manufactured by MIM and machining were obtained. Brackets were bonded to stainless steel tubes with a universal bonding technique. Each bracket was subjected to a force sufficient for total debonding by an Instron machine. Force required to debond each bracket and ARI scores were recorded. The mean shear bonding strength and ARI scores for the study sample were $3.7 \pm 1.7$ and $4.2 \pm 0.7$ respectively. The results of two sample $t$ test showed that there was no significant difference between the one-piece MIM brackets (3.5 \pm 1.7$)$ and the multi-piece brackets $(4.0 \pm 1.8)$ in mean shear bonding strength $(\mathrm{t}(128)=1.33, \mathrm{p}=0.18)$. The mean of ARI scores for the multi-piece brackets $(4.4 \pm 0.7)$ is significantly higher than the mean of ARI scores for the one-piece MIM brackets (4.0 \pm 0.7$)$ $(\mathrm{t}(128)=3.5, \mathrm{p}=0.006)$. Metal orthodontic brackets manufactured by MIM and those manufactured by machining then soldering/brazing exhibit similar shear bond strengths. However, one-piece MIM brackets showed a significantly lower ARI score than multi-piece machined brackets, indicating a higher percentage of adhesive resin left on the one-piece MIM bracket surface after debond when compared to the percentage of resin left on the multi-piece brackets at debond. This may be due to differences in pad retentive design, but further research is needed. No brackets were broken during this study; all failures occurred at the pad-adhesivemetal interface. 


\section{References:}

1.Bishara S, VonWald L, Laffoon J, Warren J. Effect of using a new cyanoacrylate adhesive on the shear bond strength of orthodontic brackets. Angle Orthod 2001;71:466-9.

2.Davis, Joseph R., ed. Stainless steels. ASM international, 1994.

3.Eliades T, Bourauel C. Intraoral aging of orthodontic materials: the picture we miss and its clinical relevance. Am J Orthod Dentofacial Orthop 2005;127:403-12.

4.Eliades, Theodore, et al. "Manufacturing of orthodontic brackets: A review of metallurgical perspectives and applications." Recent Patents on Materials Science 1.2 (2008): 135-139.

5.Iijima, Masahiro, et al. "Orthodontic brackets." Orthodontic Applications of Biomaterials. Woodhead Publishing, 2017. 75-96.

6.Kafle D, Mishra RK, Hasan MR, Saito T. A retrospective clinical audit of bracket failure among patients undergoing orthodontic therapy. International journal of dentistry. 2020;2020:8810964-8810964. doi:10.1155/2020/8810964

7.MacColl, G. A., et al. "The relationship between bond strength and orthodontic bracket base surface area with conventional and microetched foil-mesh bases." American journal of orthodontics and dentofacial orthopedics 113.3 (1998): 276-281.

8.Maijer, Rv, and D. C. Smith. "Variables influencing the bond strength of metal orthodontic bracket bases." American journal of orthodontics 79.1 (1981): 20-34.

9.Matasa CG. Direct bonding metallic brackets: where are they heading? American Journal of Orthodontics and Dentofacial Orthopedics 1992;102:552-60.

10.Millett DT, Cattanach D, Robertson M. A 5-year clinical review of bond failure with a lightcured resin adhesive. Angle Orthod 1998;68:351-6

11.Murray S, Hobson R. Comparison of in vivo and in vitro shear bond strength. Am J Orthod Dentofacial Orthop 2003;123:2-9.

12.Nucera, R. Lo Giudice, A. Bellocchio, M. Spinuzza, P. Caprioglio, A. and Cordasco, G. "Diagnostic concordance between skeletal cephalometrics, radiograph-based soft-tissue cephalometrics, and photograph-based soft-tissue cephalometrics," European Journal of Orthodontics, vol. 39, no. 4, pp. 352-357, 2017.

13.Oesterle L, Shellhart W, Belanger G. Effect of tacking time on bond strength of light-cured adhesives. J Clin Orthod 1997;31: 449-53.

14.Oesterle LJ, Shellhart WC. Effect of aging on the shear bond strength of orthodontic brackets. American journal of orthodontics and dentofacial orthopedics : official publication of 
the american association of orthodontists, its constituent societies, and the american board of orthodontics. 2008;133(5):716-720. doi:10.1016/j.ajodo.2006.04.042

15.Pickett K, Sadowsky P, Jacobson A, Lacefield W. Orthodontic in vivo bond strength: comparison with in vitro results. Angle Orthod 2001;71:141-8.

16. Sharma, J.N. "Epidemiology of malocclusions and assessment of orthodontic treatment need for the population of eastern Nepal," World Journal of Orthodontics, vol. 10, no. 4, pp. 311-316, 2009.

17.Sharma-Sayal, Seema K., et al. "The influence of orthodontic bracket base design on shear bond strength." American journal of orthodontics and dentofacial orthopedics 124.1 (2003): 7482 .

18.Singh, S. Sharma, A. Sandhu, N. and Mehta, K. "/e prevalence of malocclusion and orthodontic treatment needs in school going children of Nalagarh, Himachal Pradesh, India," Indian Journal of Dental Research, vol. 27, no. 3, pp. 317-322, 2016

19.Singh, V.P. and Sharma, A. "Epidemiology of malocclusion and assessment of orthodontic treatment need for Nepalese children," International Scholarly Research Notices, vol. 2014, Article ID 768357, 4 pages, 2014.

20.Tak, M., Nagarajappa, R. Sharda, A.J. et al., "Prevalence of malocclusion and orthodontic treatment needs among 12-15 years old school children of Udaipur, India," European Journal of Dentistry, vol. 7, no. 1, pp. S45-S53, 2013.

21.Vellappally, S. Gardens, S.J. Al Kheraif, A. et al., "the prevalence of malocclusion and its association with dental caries among 12-18-year-old disabled adolescents," BMC Oral Health, vol. 14, no. 1, p. 123, 2014.

22.Wang, Wei Nan, et al. "Bond strength of various bracket base designs." American Journal of Orthodontics and Dentofacial Orthopedics 125.1 (2004): 65-70.

23.Weir, T. (2017) Clear aligners in orthodontic treatment. Australian Dental Journal, 62: S1 58-62. doi: 10.1111/adj.12480

24.Zinelis, Spiros, et al. "Elemental composition of brazing alloys in metallic orthodontic brackets." The Angle Orthodontist 74.3 (2004): 394-399.

25.Zinelis, Spiros, et al. "Metallurgical characterization of orthodontic brackets produced by metal injection molding (MIM)." The Angle Orthodontist 75.6 (2005): 1024-1031. 